\title{
Surface-driven registration method for the structure-informed segmentation of diffusion MR images
}

\author{
Oscar Esteban ～, Dominique Zosso , Alessandro Daducci ，Meritxell Bach-Cuadra ， \\ María J. Ledesma-Carbayo ，Jean-Philippe Thiran Andres Santos
}

Keywords:

Active surfaces

Cortical parcellation

Diffusion MRI

Nonlinear registration

Segmentation

Susceptibility distortion

\begin{abstract}
A B S T R A C T
Current methods for processing diffusion MRI (dMRI) to map the connectivity of the human brain require precise delineations of anatomical structures. This requirement has been approached by either segmenting the data in native dMRI space or mapping the structural information from T1-weighted (T1w) images. The characteristic features of diffusion data in terms of signal-to-noise ratio, resolution, as well as the geometrical distortions caused by the inhomogeneity of magnetic susceptibility across tissues hinder both solutions. Unifying the two approaches, we propose regseg, a surface-to-volume nonlinear registration method that segments homogeneous regions within multivariate images by mapping a set of nested reference-surfaces. Accurate surfaces are extracted from a T1w image of the subject, using as target image the bivariate volume comprehending the fractional anisotropy (FA) and the apparent diffusion coefficient (ADC) maps derived from the dMRI dataset. We first verify the accuracy of regseg on a general context using digital phantoms distorted with synthetic and random deformations. Then we establish an evaluation framework using undistorted dMRI data from the Human Connectome Project (HCP) and realistic deformations derived from the inhomogeneity fieldmap corresponding to each subject. We analyze the performance of regseg computing the misregistration error of the surfaces estimated after being mapped with regseg onto 16 datasets from the HCP. The distribution of errors shows a $95 \% \mathrm{CI}$ of $0.56-0.66 \mathrm{~mm}$, that is below the dMRI resolution ( $1.25 \mathrm{~mm}$, isotropic). Finally, we cross-compare the proposed tool against a nonlinear $b 0$-to-T2w registration method, thereby obtaining a significantly lower misregistration error with regseg. The accurate mapping of structural information in dMRI space is fundamental to increase the reliability of network building in connectivity analyses, and to improve the performance of the emerging structure-informed techniques for dMRI data processing.
\end{abstract}

\section{Introduction}

Diffusion MRI enables the mapping of microstructure (Basser and Pierpaoli, 1996) and connectivity (Craddock et al., 2013) of the human brain in-vivo. It is generally acquired using echo-planar imaging (EPI) schemes, since they are very fast at scanning a large sequence of images called diffusion weighted images (DWIs). Each DWI is sensitized with a gradient to probe proton diffusion in a certain orientation. Subsequent processing involves describing the local microstructure with one of the available models, which range from the early diffusion tensor imaging (DTI) proposed by Basser and Pierpaoli (1996) to current models such as AMICO (accelerated microstructure imaging via convex optimization, Daducci et al., 2015). The microstructural map is then used to draw the preferential orientations of diffusion across the brain using tractography (Mori et al., 1999). Finally, a graph representing the corresponding structural network is built using the regions of a cortical parcellation as nodes and the fiber paths found by tractography as edges (Hagmann et al., 2008). The methodologies to solve reconstruction, tractography and network building require the delineation of the anatomy in the dMRI space. Moreover, current trends on reconstruction (Jeurissen et al., 2014) and tractography (Smith et al., 2012) are increasingly using structural information to improve the microstructural mapping and fiber-tracking.

Possibly, the earliest structural information incorporated to aid dMRI processing is the white matter (WM) mask used as a termination 
criteria for tractography. The standardized procedure to obtain this mask was thresholding the FA map. However, the mask and subsequent analyses are highly dependent on the threshold that is chosen (Taoka et al., 2009). To overcome the unreliability of FA thresholding, and to broaden WM segmentation to brain tissue segmentation, a large number of methods have been proposed using DWIs, the $b 0$, and DTIderived scalar maps such as FA, ADC and others (Zhukov et al., 2003; Rousson et al., 2004; Jonasson, 2005; Hadjiprocopis et al., 2005; Liu et al., 2007; Lu et al., 2008; Han et al., 2009). However, the precise segmentation of dMRI is difficult for several reasons. First, dMRI images have a resolution that is much lower than that of the imaged microstructural features. Therefore, voxels located in structural discontinuities are affected by partial voluming of the signal sources. Second, the extremely low signal-to noise ratio (SNR) and the high dimensionality of the DWIs prevent their direct use in segmentation. Third, the low contrast between gray matter (GM) and WM in the bo volume also makes it unsuitable for brain tissue segmentation.

An alternative route to segmentation in dMRI space is the mapping of the structural information extracted from anatomical MR images, such as T1w, using image registration techniques. Generally, intra subject registration of MR images of the brain involves only a linear mapping to compensate for head motion between scans. However, EPI introduces a geometrical distortion (Jezzard and Balaban, 1995) that impedes the linear mapping from the structural space. Numerous methods have been proposed to overcome this problem by incorporating information from extra MR acquisitions such as fieldmaps (Jezzard and Balaban, 1995), DWIs with a different phase-encoding (PE) scheme (Cordes et al., 2000; Chiou and Nalcioglu, 2000), or T2-weighted (T2w) images (Kybic et al., 2000; Studholme et al., 2000). These methods estimate the deformation field associated to EPI distortions and resample the DWIs onto a corrected dMRI space. The retrospective EPI correction is an active field of research yielding frequent refinements and combinations of the original methods, such as (Holland et al., 2010; Andersson et al., 2012; Irfanoglu et al., 2015). A standardized method to solve the remaining linear mapping between the corrected-dMRI and the structural spaces is bbregister (Greve and Fischl, 2009).

Here, we present a segmentation and surface-to-volume registration method called regseg, and show its usefulness in mapping anatomical information from structural space into native dMRI space to aid subsequent processing steps (reconstruction, tractography and network building using a cortical parcellation). The underlying hypothesis is that the registration and segmentation problems in dMRI can be solved simultaneously. To implement regseg we first establish an active-contours without edges (Chan and Vese, 2001) segmentation framework. A specific set of reference surfaces extracted from the same subject initialize the 3D active contours, which evolve searching for homogeneous regions in the multivariate target-image. We apply regseg to segment dMRI data by mapping a set of nested surfaces extracted from a structural image (e.g. T1w) to a bivariate target-volume comprehending the FA and ADC maps. The evolution of the surfaces is supported by a B-spline basis, optimized iteratively using a descent approach driven by shape-gradients (Jehan-Besson et al., 2003; Herbulot et al., 2006). Therefore, regseg establishes a registration framework that actually deals with the nonlinear warping induced by EPI distortions. Regseg integrates the benefits of segmentation and registration methods together and exploits the multivariate nature of dMRI data to contribute in the proposed application on three key aspects: 1 ) the surfaces are typically extracted from the T1 $w$ image of the same subject, therefore regseg does not require additional MR acquisitions to the minimal dMRI protocol in order to estimate the deformation field; 2 ) alternatively to the typical design of the processing flow, the information from the reference T1w can be precisely mapped onto the distorted dMRI space, avoiding the interpolation of the DWIs required by unwarping the diffusion data; and 3) regseg increases the geometrical accuracy of the overall process. In this paper, we first verify the functionality of the method and the regseg implementation using a set of digital phantoms, demonstrating the subvoxel accuracy in registration. Then, we evaluate regseg on real dMRI datasets, using a derivation of our instrumentation framework (Esteban et al., 2014a) which simulates known and realistic EPI distortions. We also compare regseg and a nonlinear registration method to map the $b 0$ to the corresponding T2w image of the same subject. This approach is the first step of the above-mentioned T2w-registration based (T2B) correction methods. We reproduce the settings and implementation of a widely used diffusion processing software (ExploreDTI, Leemans et al., 2009). With this comparison, we demonstrate how regseg achieves higher accuracy with the simultaneous registration and segmentation process.

\section{Methods}

\section{Registration framework and segmentation model}

Let $\boldsymbol{\Gamma}_{R}=\left\{\Gamma_{m}: m \in \mathbb{N}, m \leq N_{S}\right\}$ be the set of $N_{S}$ surfaces extracted from the undistorted $\mathrm{T} 1 \mathrm{w}$ image (the reference space $R$ ). We reformulate the segmentation of the distorted dMRI images (the moving space $M$ ) as a registration problem where we search for an underlying deformation field $U$ such that the structures in $R$ defined by $\boldsymbol{\Gamma}_{R}$ align optimally with their corresponding structures in $M$ :

$$
\begin{aligned}
U: R \subset \mathbb{R}^{n} & \rightarrow M \subset \mathbb{R}^{n} \\
\mathbf{r} & \mapsto \mathbf{r}^{\prime}=\mathbf{r}+\mathbf{u r},
\end{aligned}
$$

where $\mathbf{r}$ denotes a position in $R, \mathbf{r}^{\prime}$ is its corresponding location in $M$, and $n$ denotes the dimensionality of images. Finally, $\mathbf{u}=\mathbf{u}(\mathbf{r})$ is the displacement of every point with respect to the reference domain. The general overview of how the surfaces interact with the registration framework is presented in Fig. 1.

Cost-function derivation. In a Bayesian framework for registration (Wyatt and Noble, 2003; Pohl et al., 2006; Gass et al., 2014), the mappings $U$ in (1) are evaluated based on their posterior probability given the observed data $M$. Let $\Omega=\left\{\Omega_{l}: l \in \mathbb{N}, l \leq N_{L}\right\}$ be the set of $N_{L}$ competing

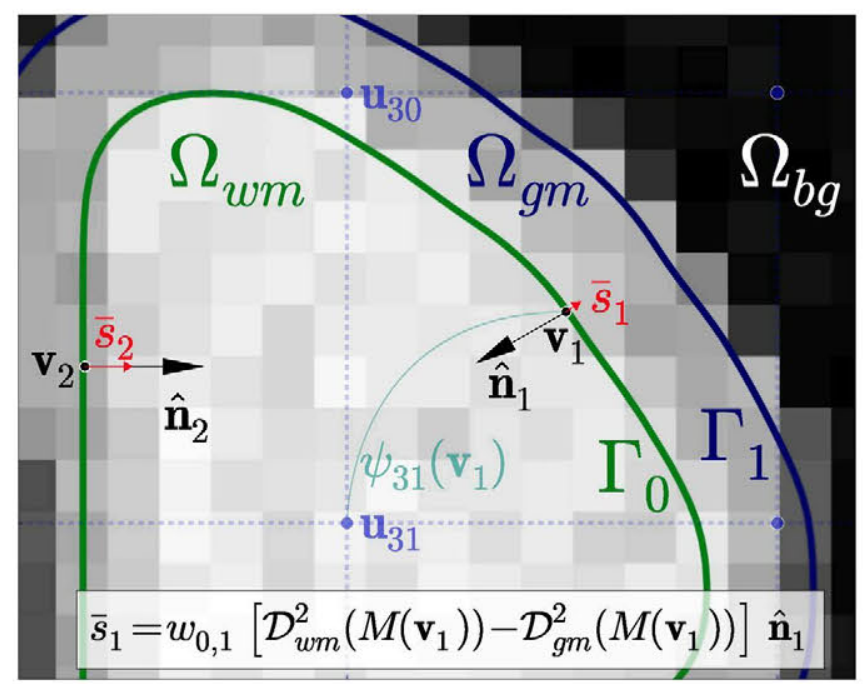

Fig. 1. The interfacing surfaces $\Gamma_{m}$ between the competing ROIs $\Omega_{b}$, play the role of active contours which drive the registration process. They evolve iteratively along the normal $\hat{\mathbf{n}}_{i}$ of the surface at each vertex $\mathbf{v}_{i}$ of the mesh. The gradient speeds $\bar{s}_{i}$ drive registration, which are computed as the disparity of the data energies with respect to the two limiting regions of $M\left(\mathbf{v}_{i}\right)$, the features of the image $M$ in the location of vertex $\mathbf{v}_{i}$. The computation of shape-gradients is developed in Appendix B. In this figure, the $\bar{s}_{1}$ derived from Eq. (A.6) is written in the lower box, with $\Omega_{w m}$ being the inner limiting region, $\Omega_{g m}$ the outer region, and $w_{0,1}$ the relative area associated with vertex $\mathbf{v}_{1}$ with respect to the total area of surface $\Gamma_{0}$. 
regions in which $M$ is partitioned by the projection of $\boldsymbol{\Gamma}_{R}$. Using Bayes' rule, the posterior likelihood is computed as:

$P(U \mid M, \mathbf{\Omega})=\frac{P(M \mid U, \boldsymbol{\Omega}) P(U)}{P(M)}$,

where $P(M \mid U, \Omega)$ is the data likelihood. Since $\boldsymbol{\Omega}$ is mapped by $U$, we simplify $P(U, \boldsymbol{\Omega})=P(U) \Rightarrow P(M \mid U, \boldsymbol{\Omega})=P(M \mid U)$. The best estimate $U$ then satisfies the maximum a posteriori criterion and it aligns $\boldsymbol{\Gamma}_{R}$ into $M$. First, we assume independence between voxels, and thus we break down the global data likelihood into a product of voxel-wise conditional probabilities:

$P(M \mid U)=\prod_{l} \prod_{\mathbf{r}^{\prime} \in \Omega_{i}} P\left(\mathbf{f}^{\prime} \mid U\right)$,

where $\mathbf{f}^{\prime}=M\left(\mathbf{r}^{\prime}\right)$ is the feature vector at the displaced position $\mathbf{r}^{\prime}(1)$ in the moving image. For convenience and because it has been shown to be an appropriate approximation (Van Leemput et al., 1999; Cuadra et al., 2005), we introduce two assumptions for each region $\Omega_{l}: 1$ ) the features are i.i.d.; and 2) they can be modeled by multivariate normal distributions $\mathcal{N}\left(\mathbf{f}^{\prime} \mid \boldsymbol{\mu}_{l}, \Sigma_{l}\right)$, with parameters $\left\{\boldsymbol{\mu}_{l}, \Sigma_{l}\right\}$ for each region $\Omega_{l}$ (Esteban et al., 2014b):

$$
\begin{aligned}
P(M \mid U) & =\prod_{l} \prod_{\mathbf{r}^{\prime} \in \Omega_{i}} \mathcal{N}\left(\mathbf{f}^{\prime} \mid \boldsymbol{\mu}_{l}, \sum_{l}\right) \\
& =\prod_{l} \prod_{\mathbf{r}^{\prime} \in \Omega_{i}} \frac{1}{\sqrt{(2 \pi)^{c}\left|\sum_{l}\right|}} e\left(-\frac{1}{2} \mathcal{D}_{l}^{2}\left(\mathbf{f}^{\prime}\right)\right),
\end{aligned}
$$

using $\mathcal{D}_{l}^{2}\left(\mathbf{f}^{\prime}\right)$ to denote the squared Mahalanobis distance of $\mathbf{f}^{\prime}$ with respect to the descriptors of region $l$ as $\mathcal{D}_{l}^{2}\left(\mathbf{f}^{\prime}\right)=\left(\mathbf{f}^{\prime}-\boldsymbol{\mu}_{l}\right)^{T} \Sigma_{l}^{-1}\left(\mathbf{f}^{\prime}-\boldsymbol{\mu}_{l}\right)$. $C$ is the number of channels comprised in the image $M$. Even though the features being segmented are not generally i.i.d., the spatial interdependency of voxels is implicitly supported by the piecewise smooth partition of the space $\boldsymbol{\Omega}$. In fact, the projection of $\boldsymbol{\Gamma}_{R}$ onto $M$ is an implicit segmentation model, for which the covariance matrix $\Sigma_{l}$ of each region is minimized. Fig. 2 shows how the joint distribution of the input images is approximated with a mixture of multivariate normal distributions, and this minimization is illustrated for the segmentation of the FA and the ADC maps of one subject.

Regularization. The smoothness of the resulting displacement field is induced by a Thikonov regularization prior:

$P(U)=\prod_{\mathbf{r}} p(\mathbf{u})=\prod_{\mathbf{r}} p_{0}(\mathbf{u}) p_{1}(\mathbf{u})$, with

$p_{0}(\mathbf{u})=\mathcal{N}\left(\mathbf{u} \mid 0, \mathbf{A}^{-1}\right)$,

$p_{1}(\mathbf{u})=\mathcal{N}\left(\nabla \mathbf{u} \mid 0, \mathbf{B}^{-1}\right)$,

which requires that the distortion and its gradient have zero mean, and variance governed by the matrices $\mathbf{A}$ and $\mathbf{B}$. Therefore, $\mathbf{A}$ and $\mathbf{B}$ are tensors that modulate the regularization, and produce deformations with preferential directions. Finally, the maximum a posteriori problem is adapted to a variational problem where we search for the minimum of an energy functional by applying $E(\mathbf{u})=-\log \{P(M \mid U) P(U)\}$ :

$$
\begin{aligned}
E(\mathbf{u})= & -\log \prod_{l} \prod_{\mathbf{r}^{\prime} \in \Omega_{l}} \mathcal{N}\left(\mathbf{f}^{\prime} \mid \boldsymbol{\mu}_{l}, \Sigma_{l}\right) p_{0}(\mathbf{u}) p_{1}(\mathbf{u})= \\
= & -\sum_{l} \int_{\Omega_{t}}\left\{\log \left[\mathcal{N}\left(\mathbf{f}^{\prime} \mid \boldsymbol{\mu}_{l}, \Sigma_{l}\right)\right]+\log \left[p_{0}(\mathbf{u}) p_{1}(\mathbf{u})\right]\right\} d \mathbf{r}^{\prime}= \\
& =\text { Const. }+\sum_{l}\left\{\int_{\Omega_{l}} \mathcal{D}_{l}^{2}\left(\mathbf{f}^{\prime}\right) d \mathbf{r}\right\}+\int_{\Omega_{l}} \frac{1}{2}\left[\mathbf{u}^{T} \mathbf{A u}+(\nabla \mathbf{u})^{T} \mathbf{B}(\nabla \mathbf{u})\right] d \mathbf{r}^{\prime} .
\end{aligned}
$$

This expression is the dual of the Mumford-Shah functional that corresponds to the framework of active contours without edges (Chan and Vese, 2001) with the anisotropic regularization term of Nagel and Enkelmann (1986).

\section{Numerical implementation}

Deformation model. Since the vertices of the surfaces $\left\{\mathbf{v}_{i}: \mathbf{v}_{i} \subset \boldsymbol{\Gamma}\right\}_{i=1 \ldots N_{V}}$ are probably located off-grid, it is necessary to derive $\mathbf{u}_{i}=\mathbf{u}\left(\mathbf{v}_{i}\right)$ from a discrete set of parameters $\left\{\mathbf{u}_{k}\right\}_{k=1 \ldots K}$. Densification is achieved using a set of associated basis functions $\psi_{k}(8)$. In our implementation, $\psi_{k}$ is a tensor-product B-spline kernel of degree three.

$\mathbf{v}_{i}^{\prime}=\mathbf{v}_{i}+\mathbf{u}_{i}=\mathbf{v}_{i}+\sum_{k} \psi_{k}(\mathbf{r}) \mathbf{u}_{k}$

Optimization. To find the minimum of the energy functional (7), we propose a gradient-descent approach with respect to the underlying deformation field using the following partial differential equation (PDE):

$\frac{\partial \mathbf{u}(\mathbf{r}, t)}{\partial t} \propto-\frac{\partial E(\mathbf{u})}{\partial \mathbf{u}_{k}}$

where $t$ is an artificial time parameter of the contour evolution and $\mathbf{u}_{k}$ are the parameters that support the estimate $U$ of the transformation at the current time point. Let us assume that the preferential directions of the displacement are aligned with the imaging axes to simplify Eq. (7) as expression (A.1) in Appendix A, and thus to compute its derivative in Eq. (9):

$$
\frac{\partial E(\mathbf{u})}{\partial \mathbf{u}_{k}}=\frac{\partial}{\partial \mathbf{u}_{k}}\left\{\sum_{l}\left[\int_{\Omega_{l}} \mathcal{D}_{l}^{2}\left(\mathbf{f}^{\prime}\right) d \mathbf{r}^{\prime}\right]+\int_{\Omega} \frac{1}{2}\left[\boldsymbol{\alpha} \cdot \mathbf{u}^{2}+\boldsymbol{\beta} \cdot(\nabla \mathbf{u})^{-2}\right] d \mathbf{r}^{\prime}\right\},
$$

where $\mathbf{u}^{\circ}=\mathbf{u}^{T} \cdot \mathbf{u}$, and $\{\alpha, \beta\}$ are the expected variances along the imaging axes of the displacement field and its gradient, respectively. Then, the data and regularization terms are split and discretized to compute their derivatives. The derivative of the data term is computed using explicit shape gradients (see Appendix B), which finally lead to obtain vertex-wise speeds of the gradient $\bar{s}_{i}$ as illustrated in Fig. 1. The shape gradient contributions $\mathbf{g}_{k}$ on the field coefficients $\mathbf{u}_{k}$ can then be computed using the expression (A.7), of Appendix B, obtaining:

$\mathbf{g}_{k}=-\sum_{i} \bar{s}_{i} \cdot \psi_{k}\left(\mathbf{v}_{i}\right) \hat{\mathbf{e}}$.

Then, introducing the analytical derivative of the regularization term, Eq. (10) is reformulated as:

$\frac{\partial E(\mathbf{u})}{\partial \mathbf{u}_{k}}=\mathbf{g}_{k}+\boldsymbol{\alpha} \cdot \mathbf{u}_{k}-\boldsymbol{\beta} \cdot\left(\Delta \mathbf{u}_{k}\right)$.

Finally, to descend this gradient, we establish a semi-implicit Euler scheme (see Supplemental Materials, section S1.3), with a step size parameter $\delta$, which we solve in the spectral domain as follows:

$\mathbf{u}_{k}^{t+1}=\mathcal{F}^{-1}\left\{\frac{\mathcal{F}\left\{\delta^{-1} \mathbf{u}_{k}^{t}-\mathbf{g}_{k}\right\}}{\mathcal{F}\left\{\left(\delta^{-1}+\boldsymbol{\alpha}\right) I-\beta \Delta\right\}}\right\}$,

where $I$ denotes the identity operator.

Implementation details and convergence. The regseg tool includes a multiresolution strategy on the free-form deformation field. Registration pyramids are created by setting the spacing between the control points of the B-spline basis functions for each level of the multiresolution strategy. As a rule of thumb, for a $\delta=1.0$, both $\alpha$ and $\boldsymbol{\beta}$ will typically be in the range $[0.0,1.0]$. The parameters used $(\delta, \alpha, \beta$, 
A. OPTIMIZATION OF THE SEGMENTATION MODEL

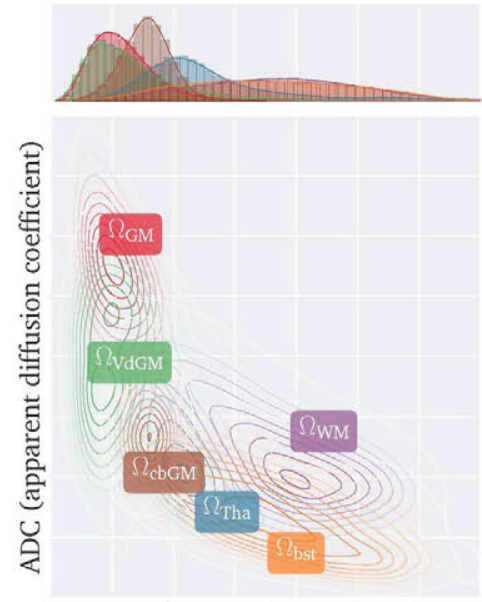

FA (fractional anisotropy)

Reference: segmentation by the surfaces extracted in T1w and original (undistorted) diffusion data.

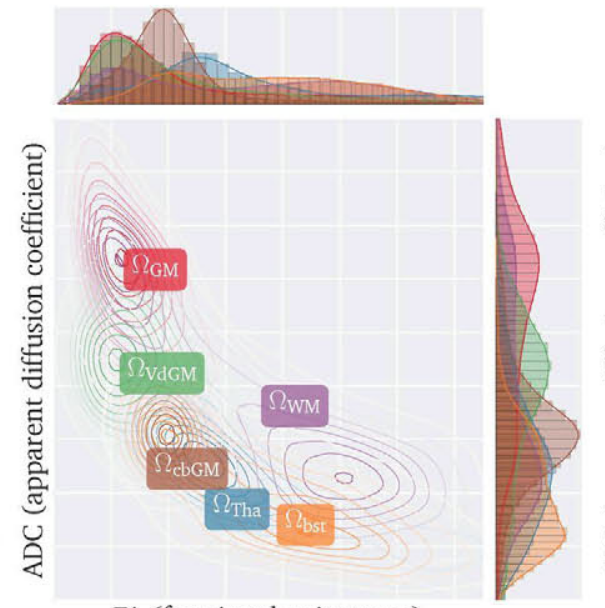

FA (fractional anisotropy)

Before registration, the contours from the T1w are not aligned with the distorted diffusion data.

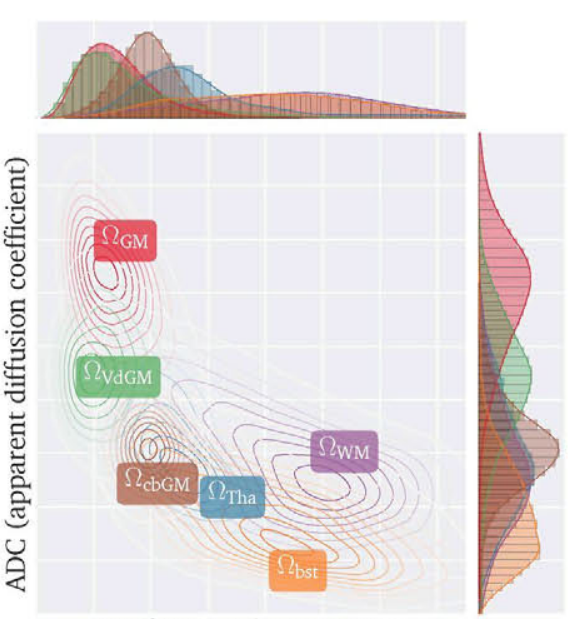

FA (fractional anisotropy)

After registration, the contours are now aligned with the distorted diffusion data.

\section{B. DETAIL OF TISSUE-WISE JOINT-DISTRIBUTIONS}

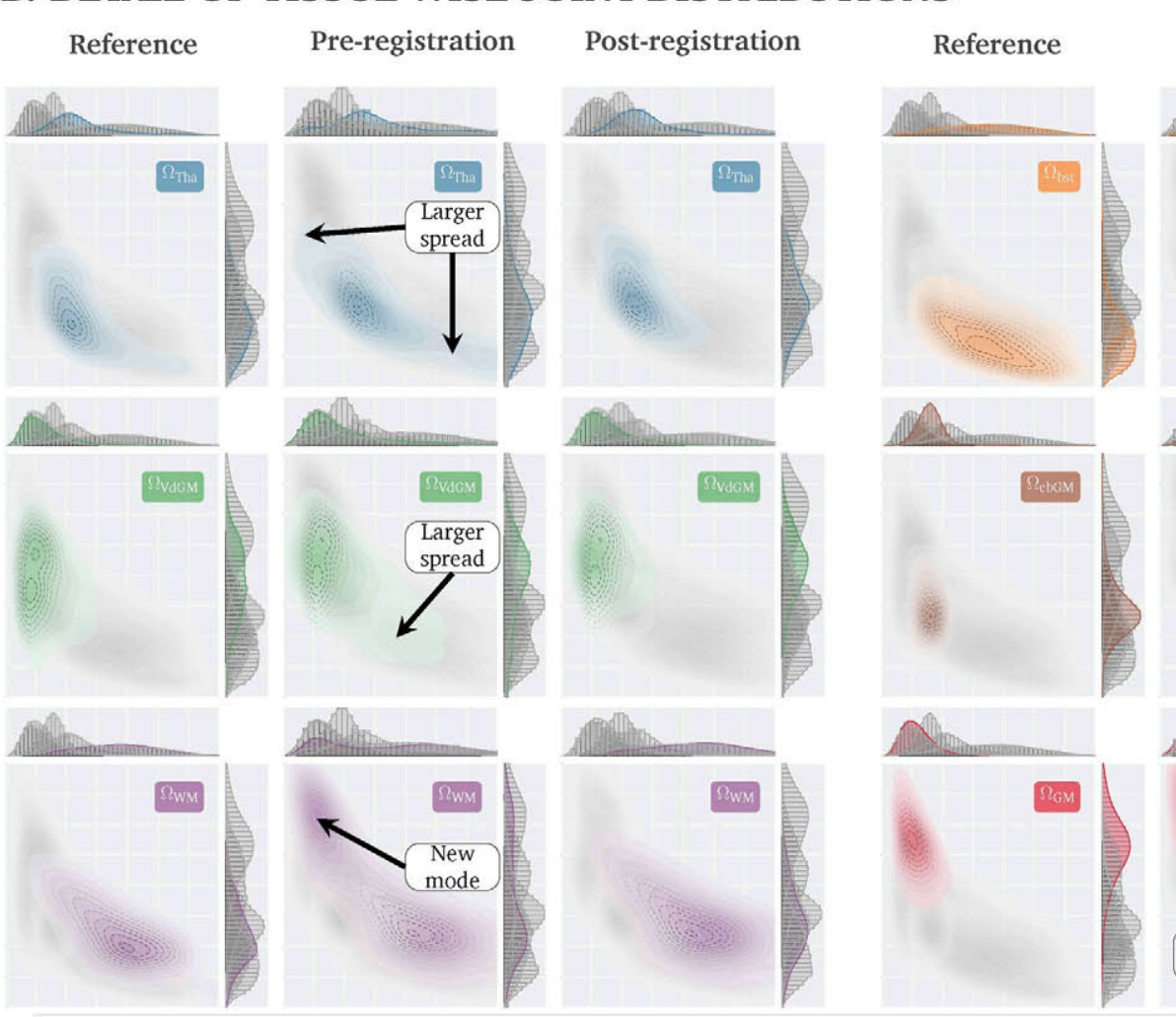

THE PROPOSED SEGMENTATION MODEL comprehends the following six homogeneous regions, in hierarchical order: 1) thalamu structures $\Omega_{\mathrm{V} \text { aic }}$; 3) cerebral white matter $\Omega_{\mathrm{WM}}$; 4) brain stem and cerebellar white matter $\Omega_{\mathrm{g}}$; 5) cerebellar gray matter $\Omega_{\text {. }}$

\section{Pre-registration Post-registration}
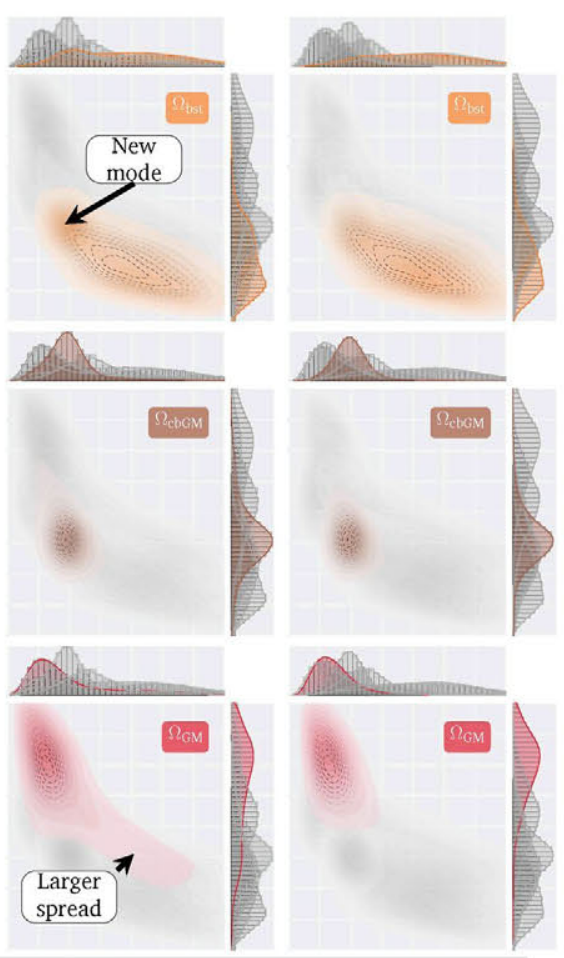

2) ventricular system and deep gray matter ; and 6) cortical gray matter $\Omega_{\mathrm{GM}}$.

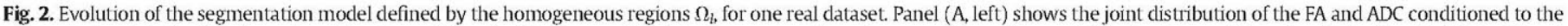

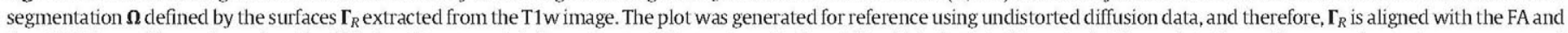

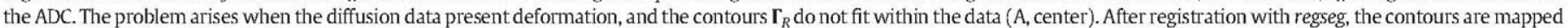

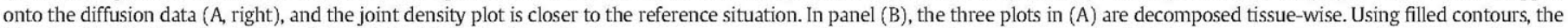

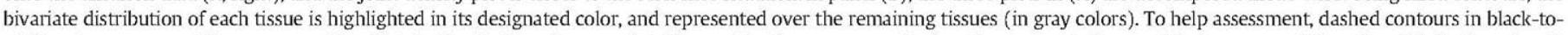

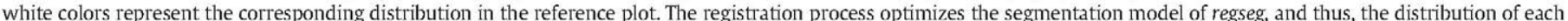

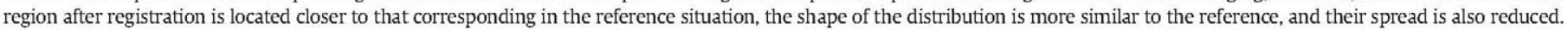

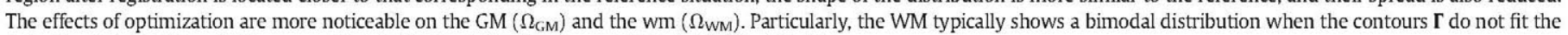
data. The plots in (A) and (B) are provided at full-size in the Supplemental Materials, Figs. S8, S9. 


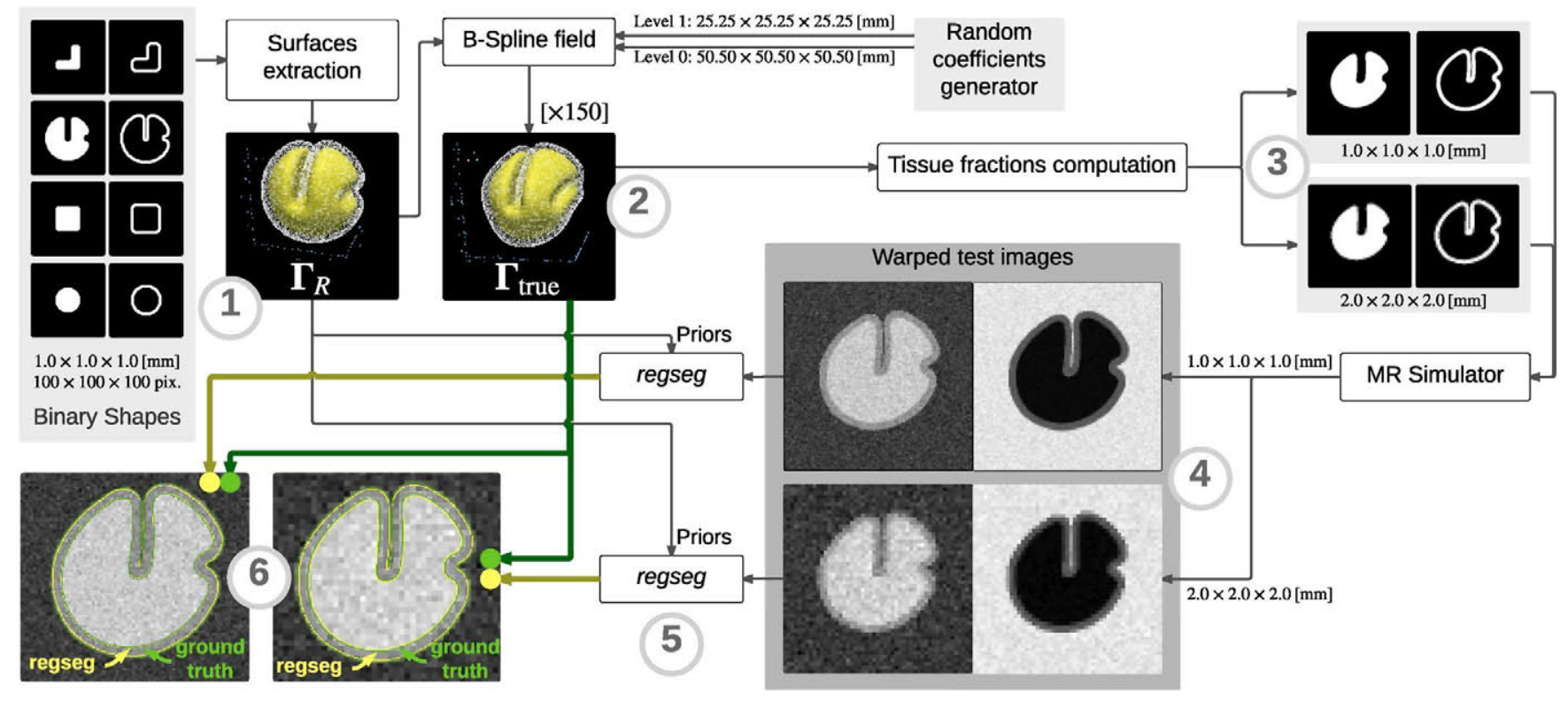

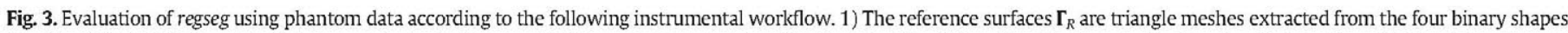

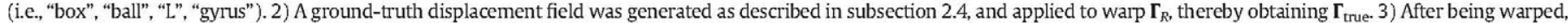

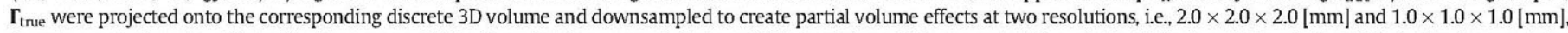

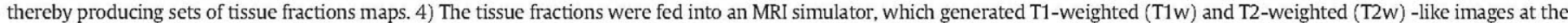

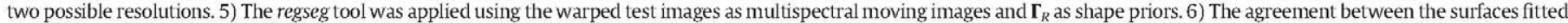
by regseg $\left(\boldsymbol{\Gamma}_{\text {test }}\right)$ and $\boldsymbol{\Gamma}_{\text {true }}$ were assessed visually using automatically generated visual reports and quantitatively with the Hausdorff distance between the corresponding surfaces.

the B-spline grid resolutions, and target image smoothing), the implementation details, and other features such as the sparse matrix approach to fast interpolation are discussed in the Supplemental Materials, section S1.

\section{Evaluation protocol}

In order to assess the performance of regseg, we defined the following general evaluation protocol: 1) Extract the set of undistorted surfaces $\boldsymbol{\Gamma}_{R} ; 2$ ) Compute a ground-truth field of displacements $U_{\text {true }}$, which is applied to generate warped images $(M)$ for segmentation; 3) Execute regseg with $\boldsymbol{\Gamma}_{R}$ and use the warped data as inputs; and 4) Perform a visual assessment and compute the error metrics.

A first proof of concept is introduced to demonstrate regseg in digital phantoms with simple geometries, using $U_{\text {true }}$ without directional restrictions. Then, regseg is evaluated in a framework using undistorted dMRI datasets, and $U_{\text {true }}$ is derived from the corresponding inhomogeneity fieldmap of the subject. Therefore, the deformation field is nonzero only in the phase-encoding (PE) axis, and reproduces a real EPI distortion. The adaptation of the evaluation protocol to the simulated phantoms and the real data is explained in the following sections.

\section{Simulated phantoms}

The workflow required to simulate the digital phantoms and to assess the performance of regseg with them is presented in Fig. 3. A set of four binary objects (i.e. "box", "ball", "L", and "gyrus") was generated by combining the binarization of analytical shapes and mathematical morphology. The reference surfaces $\boldsymbol{\Gamma}_{R}$ were extracted from the binary shapes using FreeSurfer tools (Fischl, 2012). The ground-truth distortion was generated using a chain of two displacement fields supported by grids of B-spline basis functions. The coefficients of the basis functions were generated randomly for both levels in their three dimensions. The three components of the displacements $\mathbf{u}=\left(u_{d}\right)$ were bounded above by $40 \%$ of the separation between the control points at each level to obtain diffeomorphic transforms after concatenation (Rueckert et al., 2006). The first deformation field was applied to generate large warpings with control points separated by $50.50 \mathrm{~mm}$ in the three dimensions $\left(u_{d} \leq 20.20 \mathrm{~mm}\right)$. With the second warping, we aimed to obtain a field with smoothness close to that found in a typical distortion field of dMRI data (Irfanoglu et al., 2011). Therefore, the control points were separated by $25.25 \mathrm{~mm}\left(u_{d} \leq 10.10 \mathrm{~mm}\right)$. After generating the ground-truth deformation, the original surfaces were warped by interpolating the displacement field at each vertex. The warped surfaces $\boldsymbol{\Gamma}_{\text {true }}$ were binarized to generate tissue fractions at low $(2.0 \times 2.0 \times 2.0[\mathrm{~mm}])$ and high $(1.0 \times 1.0 \times 1.0[\mathrm{~mm}])$ resolutions. Using an MRI simulator (Caruyer et al., 2014), we synthesized T1w (TE/ $\mathrm{TR}=10 / 1500 \mathrm{~ms})$ and T2 $\mathrm{w}$ images $(\mathrm{TE} / \mathrm{TR}=90 / 5000 \mathrm{~ms})$, which corresponded to each phantom type, with each at two resolutions (1.0 $\mathrm{mm}$ and $2.0 \mathrm{~mm}$ isotropic). The field of view at both resolutions was $100 \times 100 \times 100[\mathrm{~mm}]$. Next, regseg was applied to map $\boldsymbol{\Gamma}_{R}$ onto the warped phantoms to obtain the registered surfaces $\left(\hat{\boldsymbol{\Gamma}}_{\text {test }}\right)$. To quantify the misregistration error, we computed the Hausdorff distance between $\hat{\boldsymbol{\Gamma}}_{\text {test }}$ and $\boldsymbol{\Gamma}_{\text {true }}$ using (Commandeur et al., 2011). In total, 1200 experiments (four phantom types $\times 150$ random warpings $\times$ two resolutions) were performed according to the workflow illustrated in Fig. 3.

Segmentation model and settings. The segmentation model of the phantoms is implicitly defined: all phantoms comprehend an inner surface enclosing a uniform WM-like region, and an outer surface wrapping a GM-like layer. The outside is filled with uniform background (see Fig. 3). All the experimental settings used for the phantoms are made available in a unique configuration file. ${ }^{1}$

\section{Real datasets}

The experimental framework for the real datasets is presented in Fig. 4, which extends our previous evaluation (Esteban et al., 2014a) of distortions using dMRI phantoms.

Data. To evaluate regseg using real dMRI data obtained from human brains, we collected 16 subjects from the Q3 Release of the $\mathrm{HCP}$ 


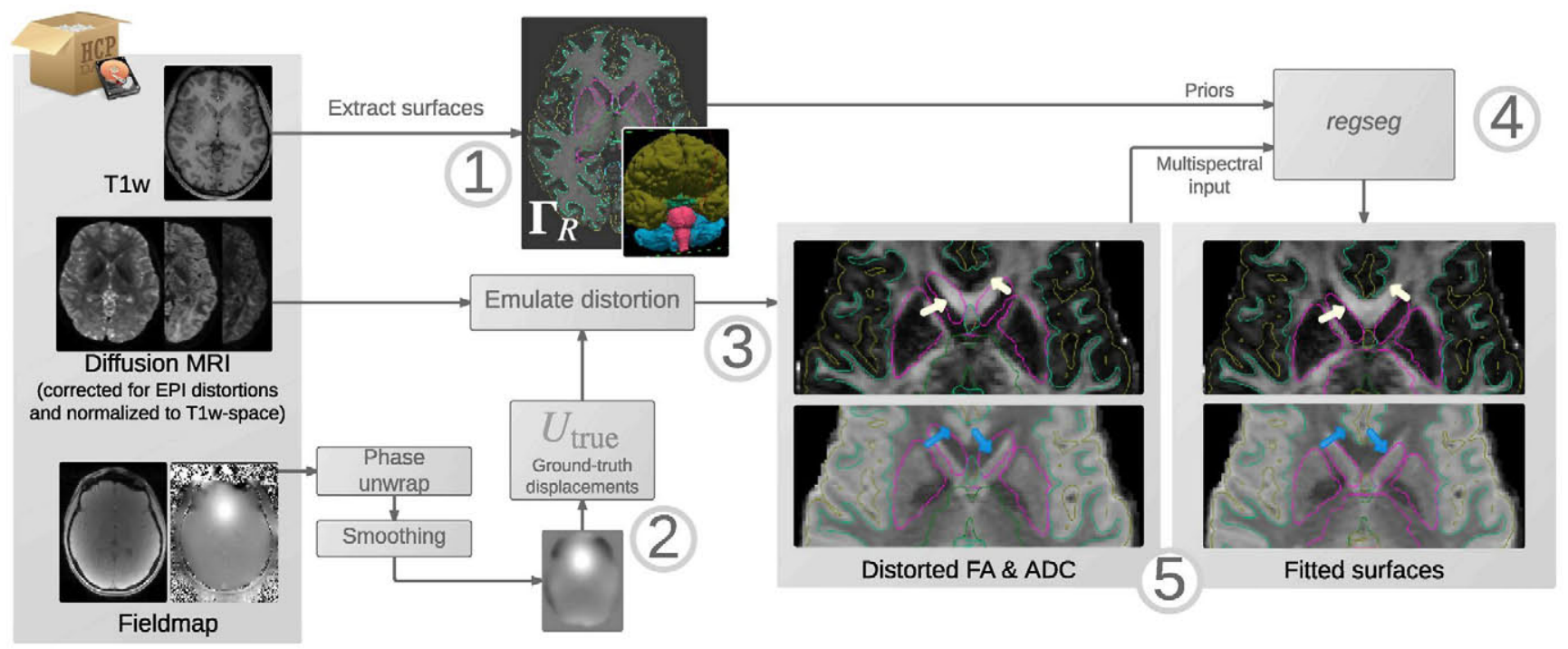

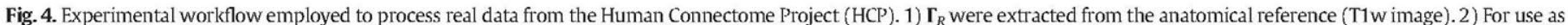

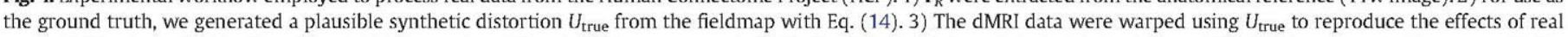

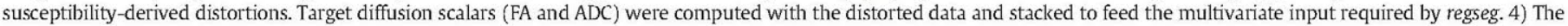

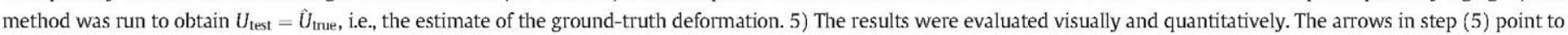

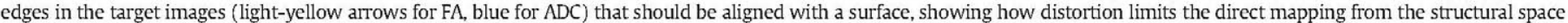
in which the contours are defined.

database. The original acquisitions are released within "unprocessed" packages, whereas the "minimally preprocessed" packages contain the corresponding images after some processing (correction for several artifacts, brain-extraction, spatial normalization, etc.). We refer the reader to Van Essen et al. (2012) for exact details of the acquisition parameters and Glasser et al. (2013) for the preprocessing issues. These datasets comprise a large set of images, including T1w, T2w, and multi-shell dMRI images. Since we obtained the dMRI data from the minimally preprocessed package, these images are corrected for EPI distortions and spatially normalized in T1w space. In the Q3 Release of the HCP, the dMRI session includes six runs, two runs for each of three different gradient tables, and each table is acquired once with right-to-left and left-to-right encoding polarities, respectively. Then, the diffusion datasets with opposed polarities are corrected for susceptibility distortions using the TOPUP tool (Andersson et al., 2003) before producing the released "minimally preprocessed" data.

Segmentation model. Based on our experience and previous studies (Ennis and Kindlmann, 2006), we defined the moving image as a stack of the FA and ADC maps derived from dMRI data. After evaluating several alternative models, we empirically defined a partition $\boldsymbol{\Omega}$ according to the following six regions: 1$)$ thalamus $\left(\Omega_{\mathrm{Tha}}\right) ; 2$ ) ventricular system and deep GM structures $\left.\left(\Omega_{\mathrm{VdGM}}\right) ; 3\right)$ cerebral WM $\left(\Omega_{\mathrm{WM}}\right) ; 4$ ) brain stem and cerebellar WM $\left.\left(\Omega_{\mathrm{bst}}\right) ; 5\right)$ cerebellar GM $\left(\Omega_{\mathrm{cbGM}}\right)$; and 6$)$ cortical GM $\left(\Omega_{\mathrm{GM}}\right)$. Using tools in FreeSurfer and appropriate selections of labels in the aparc segmentation released with the HCP data, we extracted the $\boldsymbol{\Gamma}_{R}$ set for the reference surfaces. The segmentation model corresponding to this partition is shown in Fig. 2 and discussed in greater detail in the Supplemental Materials, Section S4.

Ground-truth generation. Realistic deformation was achieved by generating displacement fields that satisfy the theoretical properties of distortion. The displacements along the PE axis of the dMRI image are related to the local deviation of the field $\Delta B_{0}(\mathbf{r})$ from its nominal value $B_{0}$ (Jezzard and Balaban, 1995), as follows:

$u_{\mathrm{PE}}=\frac{\gamma T_{a c q} S_{\mathrm{PE}}}{2 \pi} \Delta B_{0}(\mathbf{r})[\mathrm{mm}]$, where $\gamma$ is the gyromagnetic ratio, $T_{a c q}$ is the readout time, and $S_{\mathrm{PE}}$ is the voxel size along PE. Certain MRI sequences are designed to estimate $\Delta B_{0}$, thereby obtaining the so-called fieldmap. We derived the deformation $U_{\text {true }}$ from the fieldmap image released with the corresponding packages of each dataset in the HCP. The fieldmap was unwrapped ${ }^{2}$ and smoothed before applying Eq. (14). Next, the original dMRI was warped using the resulting displacement field and fed into a pipeline to process the corresponding DTI, thereby computing the derived scalars of interest (FA and ADC) using MRtrix (Tournier et al., 2012).

Metric assessment. Initially, we investigated the appropriateness of the segmentation model. For five test datasets, we uniformly sampled the space of distortions $\hat{U}=\epsilon \cdot U_{\text {true }}=\mathbf{r}+\varepsilon u_{\mathrm{PE}}$ (with $\epsilon \in[-1.1,1.1]$ and $u_{\mathrm{PE}}$ from Eq. (14)), and we evaluated the data term of the cost function in Eq. (7). The minimum of the cost function (subsection 2.1) was consistently located at $\epsilon=0.0$ (the ground-truth) for all of the cases (Supplemental Materials, Fig. S2).

Settings. Regseg accepts an affine mapping from surface-space to the dMRI data as initialization. However, the images provided by the HCP are already spatially normalized. Therefore, the initial estimation of distortion is zero in this experiment. Since the distortion $U_{\text {true }}$ is aligned along the PE direction ( $y$-axis in our settings), regseg was configured to allow nonzero displacements only on that corresponding direction. For the experiments on real data, regseg established a multi-resolution pyramid of B-spline functions, with control points distributed on grids of the following spacings: $40 \times 100 \times 40[\mathrm{~mm}]$ for the first (coarser) level, $30 \times 30 \times 30[\mathrm{~mm}]$ for the second level, and $20 \times 30 \times 10[\mathrm{~mm}]$ in the third level. Only the first and second levels included Gaussian smoothing of the target image ( $O=[2.0,0.5] \mathrm{mm}$, respectively). The actual choices of the parameter settings are publicly distributed with the source code for the experiments. ${ }^{3}$ These settings were obtained manually, driven by the feedback obtained from the post-registration convergence reports (like that found in Supplemental Materials, section S1.3). 
We released regseg along with the tool to generate such convergence reports.

Cross-comparison. A dual workflow to the general evaluation used for regseg (Fig. 4), was employed to integrate the alternate $\mathrm{T} 2 \mathrm{~B}$ registration scheme. We reproduced the solution and settings provided with ExploreDTI (Leemans et al., 2009), which is a widely used toolkit for tractography analysis of DTI. ExploreDTI internally employs elastix (Klein et al., 2010) to perform registration. The deformation field is correspondingly restricted to the PE direction. The settings file for elastix is also available. ${ }^{4}$ In this registration scheme, the T2w image is the reference and the $b 0$ plays the role of moving image. Therefore, the transform is defined in the coordinate system of the T2w image, and for each point in this space it provides the location of the corresponding feature in the $b 0$ image. Since the surfaces are defined in the T2w-reference-space, their coordinates can be mapped to the $b 0$ space using this transform, obtaining the distorted surfaces corresponding to the b0-to-T2w registration.

Error measurement. Distortion only occurs along the PE axis of the image, so we computed the surface warping index (sWI) as the areaweighted distance between the corresponding vertices of $\boldsymbol{\Gamma}_{\text {true }}$ and their estimate obtained by the method under the test $\hat{\boldsymbol{\Gamma}}_{\text {test }}$ :

$s W I=\frac{1}{\sum_{i} a_{i}} \sum_{i}^{N_{v}} a_{i}\left\|\mathbf{v}_{i}-\hat{\mathbf{v}}_{i}\right\|$,

where $\mathbf{v}_{i} \subset \boldsymbol{\Gamma}_{\text {true }}$ are the locations of the total $N_{V}$ vertices, $a_{i}$ is the area corresponding to each vertex $\mathbf{v}_{i}$, and $\hat{\mathbf{v}}_{i} \subset \hat{\boldsymbol{\Gamma}}_{\text {test }}$ are the recovered locations that correspond to $\mathbf{v}_{i}$. In practice, we only report the sWI for three surfaces $\left(\left\{\Gamma_{\mathrm{VdGM}}, \Gamma_{\mathrm{WM}}, \Gamma_{\text {pial }}\right\}\right)$ of crucial interest in whole-brain tractography. The sWI is always computed on the dMRI space.

\section{Results}

\section{Verification and validation using digital phantoms}

The results summarized in Fig. 5 demonstrated that the accuracy was high and below the image resolution. Panel B on Fig. 5 shows the violin plots for each model type corresponding to the two sets of resolutions for the generated phantoms. In order to relate the average misregistration error to the resolution of the moving image, we proceeded as follows. First, we confirmed that the vertex-wise error distributions were skewed by using the Shapiro-Wilk's test of normality. All of the distributions of errors in the tests (four phantom types $x$ two resolutions) were nonnormal with $p<0.001$. Consequently, we used the nonparametric Wilcoxon signed-rank test with the Bonferroni correction for multiple comparisons ( $N=150$, for each phantom type). The average errors were significantly lower than the voxel size with $p<(0.001 / 150)$ in all tests (four phantom types $\times$ two resolutions). Statistical tests might not be sufficiently conclusive, so we also computed the confidence intervals, as shown in Table 1.

\section{Evaluation using real datasets and cross-comparison}

Finally, we compared the performance of regseg with that of the standard T2B method. Summary reports for visual assessment of the 16 cases are included in the Supplemental Materials, section S5. In Fig. 6 , box $A$, the visual report is shown for one subject. We computed the sWI (15) of every surface after registration using both the regseg and T2B methods. Finally, to compare the results, we performed Kruskal-Wallis H-tests (a nonparametric alternative to ANOVA) on the warping indices for the three surfaces of interest selected in section

\footnotetext{
${ }^{4}$ https://github.com/oesteban/RegSeg/blob/master/Scripts/pyacwereg/data/t2b_ elastix_y.txt.
}

$2.5\left(\Gamma_{\mathrm{VdGM}}, \Gamma_{\mathrm{WM}}, \Gamma_{\text {pial }}\right)$. All of the statistical tests showed that the error distributions obtained with regseg and T2B were significantly different, and the violin plots in box B of Fig. 6 demonstrate that the errors were always larger with T2B. We also show the $95 \%$ CIs of the sWI for these surfaces (Table 2). The aggregate $\mathrm{CI}$ for regseg was $0.56-0.66[\mathrm{~mm}]$, whereas the T2B method yielded an aggregate $\mathrm{CI}$ of $2.05-2.39$ [mm]. The results of the statistical tests and the Cls are summarized in Table 2.

\section{Discussion}

We present regseg, a simultaneous segmentation and registration method that maps a set of nested surfaces into a multivariate targetimage. The nonlinear registration process evolves driven by the fitness of the piecewise-smooth classification of voxels in the target volume imposed by the current mapping of the surfaces. We propose regseg to map anatomical information extracted from $\mathrm{T} 1 \mathrm{w}$ images into the corresponding dMRI of the same subject. Previously, joint segmentation and registration has been applied successfully to other problems such as longitudinal object tracking (Paragios, 2003) and atlas-based segmentation (Gorthi et al., 2011). The most common approach involves optimizing a deformation model (registration) that supports the evolution of the active contours (segmentation), like Paragios (2003); Yezzi et al. (2003). Regseg can be seen as a particular case of atlas-based segmentation-registration methods, replacing the atlas by the structural image of the subject (structure-informed segmentation). The main difference of atlas-based segmentation and the application at hand is the resolution of the target image. Atlas-based segmentation is typically applied on structural and high-resolution images. A comprehensive review of joint segmentation and registration methods applied in atlasbased segmentation is found in (Gorthi et al., 2011). They also propose a multiphase level-set function initialized from a labeled atlas to implement the active contours that drive the atlas registration. Alternatively, regseg implements the active contours with a hierarchical set of explicit surfaces (triangular meshes) instead of the multiphase level sets, and registration is driven by shape-gradients (Herbulot et al., 2006). As an advantage, the use of explicit surfaces enables segmenting dMRI images with accuracy below voxel size.

An important antecedent of regseg is bbregister (Greve and Fischl, 2009). The tool has been widely adopted as the standard registration method to be used along with the EPI correction of choice. It implements a linear mapping and uses 3D active contours with edges to search for intensity boundaries in the $b 0$ image. The active contours are initialized using surfaces extracted from the T1w using FreeSurfer (Fischl, 2012). To overcome the problem of nonlinear distortions, bbregister excludes from the boundary search those regions that are typically warped. Indeed, the distortion must be addressed separately because it is not supported by the affine transformation model. Conversely, the deformation model of regseg is nonlinear and the active contours are without edges (Chan and Vese, 2001) since the FA and ADC maps do not present steep image gradients (edges) but the anatomy can be identified by looking for piece-wise smooth homogeneous regions.

Recently, Le Guyader and Vese (2011) proposed a simultaneous segmentation and registration method in 2D using level sets and a nonlinear elasticity smoother on the displacement vector field, which preserves the topology even with very large deformations. Regseg includes an anisotropic regularizer for the displacement field described by Nagel and Enkelmann (1986). This regularization strategy conceptually falls in the midway between the Gaussian smoothing generally included in most of the existing methodologies, and the complexity of the elasticity smoother of Le Guyader and Vese (2011). Other minor features that differ from current methods in joint segmentation and registration are the support of multivariate target-images and the efficient computation of the shape-gradients implemented with sparse matrices.

We verified that precise segmentation and registration of a set of surfaces into multivariate data is possible on digital phantoms. We randomly deformed four different phantom models to mimic three 


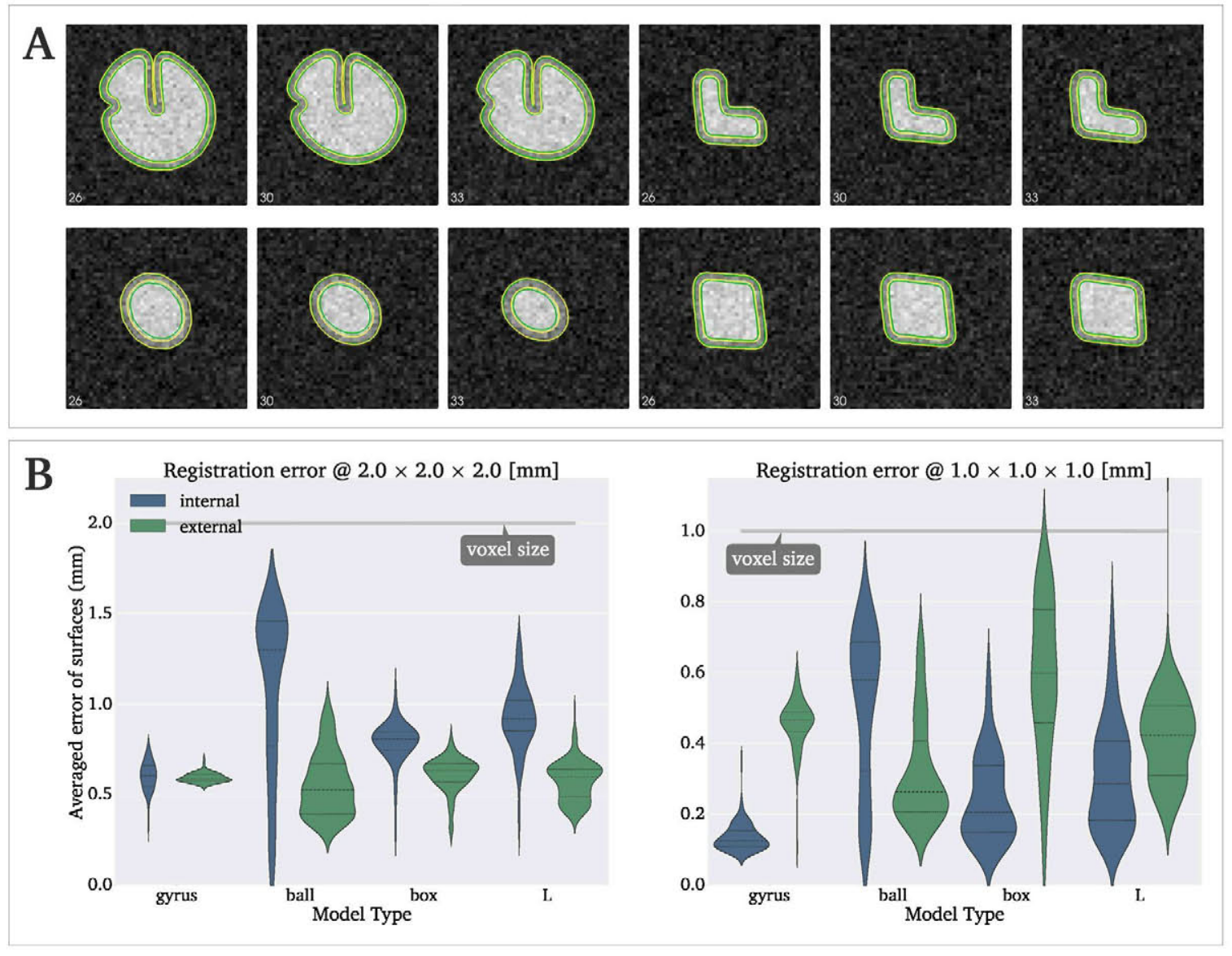

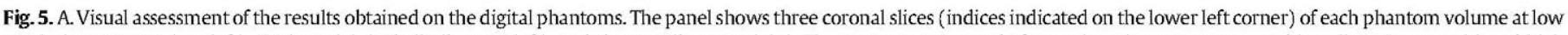

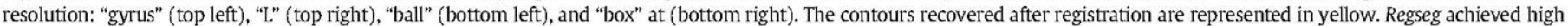

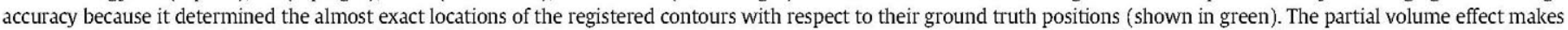

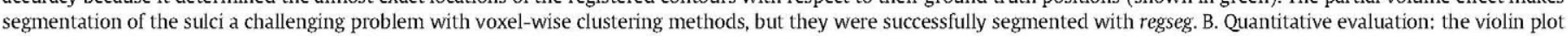

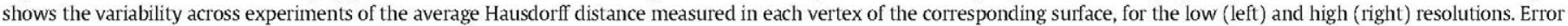
averages were consistently below the size of the voxel.

homogeneous regions (WM, GM, and cerebrospinal fluid) and we used them to simulate $\mathrm{T} 1 \mathrm{w}$ and $\mathrm{T} 2 \mathrm{w}$ images at two resolution levels. We measured the Hausdorff distance between the contours projected using the ground-truth warping and the estimations found with regseg. We concluded that the errors were significantly lower than the voxel size. We also assessed the $95 \%$ confidence interval $(\mathrm{Cl})$, which yielded an aggregate interval of $0.64-0.66$ [mm] for the low resolution phantoms ( $2.0 \mathrm{~mm}$ isotropic voxel) and $0.34-0.38[\mathrm{~mm}]$ for the high resolution phantoms (1.0 mm isotropic). Therefore, the error was bounded above by half of the voxel size. The distributions of errors along surfaces varied importantly depending on the shape of the phantom (see Fig. 5B). The misregistration error of the "gyrus" phantom showed a much lower spread than that for the other shapes. We argue that the symmetry of those other shapes posed difficulties in driving the contours towards the appropriate region due to sliding displacements between the surfaces and their ground-truth position. The effect is not detectable by the active contours framework, but it is controllable

Table 1

The distributions of vertex-wise Hausdorff distances between the ground-truth surfaces and their corresponding estimates obtained with regseg presented a $95 \% \mathrm{Cl}$ below the half-voxel size for all of the phantom types. The Cls were computed by bootstrapping using $10^{4}$ samples, with the median as the location statistic.

\begin{tabular}{lccccc}
\hline Res. & "Gyrus" & "Ball" & "Box" & "L" & Aggreg. \\
\hline $1.0 \mathrm{~mm}$ & $0.18-0.38$ & $0.31-0.45$ & $0.34-0.42$ & $0.34-0.40$ & $0.34-0.38$ \\
$2.0 \mathrm{~mm}$ & $0.59-0.60$ & $0.65-0.76$ & $0.68-0.71$ & $0.67-0.77$ & $0.64-0.66$ \\
\hline
\end{tabular}

increasing the regularization constraints. When regseg is applied on real datasets, this surface sliding is negligible for the convoluted nature of cortical surfaces and the directional restriction of the distortion.

We evaluated regseg in a real environment using the experimental framework presented in Fig. 4. We processed 16 subjects from the $\mathrm{HCP}$ database using both regseg and an in-house replication of the T2w registration based (T2B) method. Regseg obtained a high accuracy, with an aggregate $95 \% \mathrm{Cl}$ of $0.56-0.66[\mathrm{~mm}]$, which was below the voxel size of $1.25 \mathrm{~mm}$. The misregistration error that remained after regseg was significantly lower $(p<0.01)$ than the error corresponding to the T2B method according to Kruskal-Wallis H-tests (Table 2). Visual inspections of all the results (Supplemental Materials, section S5) and the violin plots in Fig. 6 confirmed that regseg achieved higher accuracy than the T2B method in our settings. We carefully configured the T2B method using the same algorithm and the same settings employed in a widely-used tool for dMRI processing. However, cross-comparison experiments are prone to the so-called instrumentation bias (Tustison et al., 2013). Therefore, these results did not prove that regseg is better than $\mathrm{T} 2 \mathrm{~B}$, but indicated that regseg is a reliable option in this application field. Finally, we also proposed a piecewise-smooth segmentation model defined by a selection of nested surfaces to partition the multispectral space comprehending the FA and the ADC maps and ultimately identify anatomical structures in dMRI space. We also demonstrated the smoothness of the objective function on five of the real datasets (Supplemental Materials, Fig. S2), taking advantage of the directional restriction of possible distortions. However, regseg requires densely sampled surfaces to ensure the convergence. Using the digital phantoms, we 

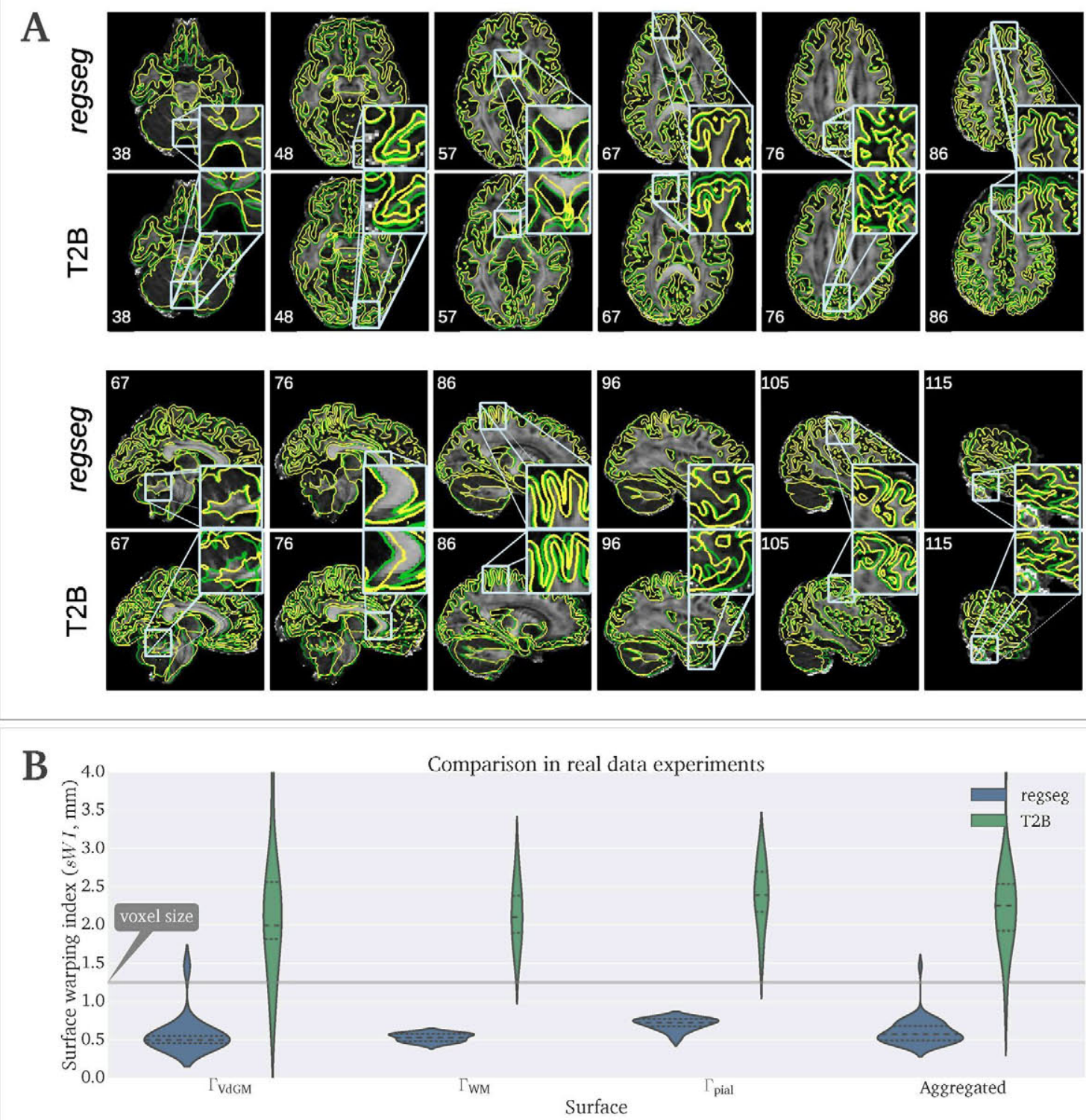

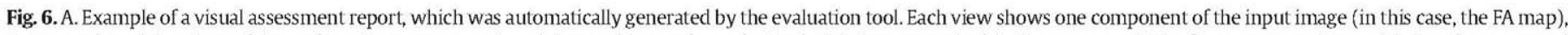

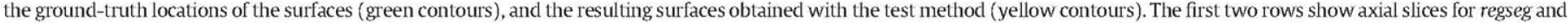

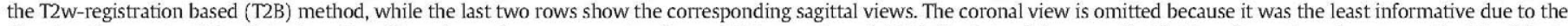

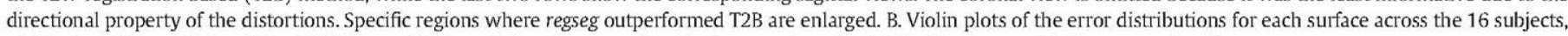
which show the voxel size of the dMRI images $(1.25 \mathrm{~mm})$, thereby supporting the improved results obtained by regseg with the proposed settings.

severely decimated the surfaces by a large factor. These surfaces introduced a bias which displaced the zero of the gradients from the minimum of the objective function impeding the convergence.

The proposed application of the method in the task of identifying structural information in dMRI images is an active field of research (Jeurissen et al., 2015). Current processing of dMRI involved in the connectome extraction and other applications (such as tract-based spatial statistics -TBSS- or surgical planning) require a precise segmentation of the anatomical structures in the diffusion space. Some examples of these processing tasks are the structure-informed reconstruction of dMRI data (Jeurissen et al., 2014; Daducci et al., 2015), the anatomically constrained tractography (Smith et al., 2012), and the imposition of the cortical parcellation mapped from the $\mathrm{T} 1 \mathrm{w}$ image (Hagmann et al., 2008). The problem was firstly addressed using image segmentation approaches in the native diffusion space, without definite and compelling results. With the introduction of retrospective correction methods for the EPI distortions and image registration approaches, the task has been typically solved in a two-step approach. First, the DWIs are corrected for EPI distortions by estimating the nonlinear deformation field from extra MR acquisitions (Jezzard and Balaban, 1995; Chiou and Nalcioglu, 2000; Cordes et al., 2000; Kybic et al., 2000). Second, mapping the structural information from the corresponding T1w image using a linear registration tool like bbregister (Greve and Fischl, 2009). The current activity on improving correction methods (Irfanoglu et al., 2015) and the comeback of segmentation of dMRI in its native space (Jeurissen et al., 2015) proof the open interest of this application. Regseg addresses this joint problem in a single step and it does not require any additional acquisition other than the minimal protocol comprehending only T1w and dMRI images. This situation is commonly found in historical datasets. 
Table 2

Statistical analysis of results obtained using 16 real datasets from the HCP, which shows that regseg performed better than the alternative $\mathrm{T} 2 \mathrm{w}$-registration based (T2B) method. The distribution of the errors computed for the surfaces of interest $\left(\Gamma_{\mathrm{VdGM}}, \Gamma_{\mathrm{WM}}, \Gamma_{\text {pial }}\right)$ and the aggregate of all surfaces (Aggreg. column) had lower $95 \% \mathrm{CIs}$ with regseg. The CIs in this table were computed by bootstrapping using the mean as the location statistic and with $10^{4}$ samples. The Kruskal-Wallis H-tests indicated that there was a significant difference between the results obtained using regseg and the T2B method.

\begin{tabular}{llcccc}
\hline & & $\Gamma_{\text {VdGM }}$ & $\Gamma_{\text {WM }}$ & $\Gamma_{\text {pial }}$ & Aggreg. \\
\hline $\mathrm{CI}$ & regseg & $0.50-0.78$ & $0.50-0.55$ & $0.66-0.73$ & $0.56-0.66$ \\
& T2B & $1.78-2.58$ & $1.94-2.36$ & $2.16-2.58$ & $2.05-2.39$ \\
\multirow{2}{*}{ H-tests } & p-value & $4.1 \cdot 10^{-6}$ & $2.3 \cdot 10^{-6}$ & $2.3 \cdot 10^{-6}$ & $1.8 \cdot 10^{-16}$ \\
& H-stat & 21.20 & 22.31 & 22.31 & 67.85 \\
\hline
\end{tabular}

We envision regseg to be integrated in diffusion processing pipelines, after a preliminary DTI computation and before anatomically-informed reconstruction and tractography methods. Since the structural information is projected into the native space of dMRI, these two processes and the matrix building task can be performed on the unaltered dMRI signal (i.e. without resampling data to an undistorted space). For analyses other than connectivity, like TBSS, the deformation estimated by regseg can be used to map the tracts into structural space. Even though we apply regseg to the problem of susceptibility distortion, it is not a distortion correction method, but rather a surface alignment method. In fact, the distortions are not corrected in the EPI data. Therefore, we suggest here to perform the reconstruction and tractography processes in the original (distorted) diffusion data. Regseg allows to avoid resampling and/or unwarping of the diffusion signal because the structural information necessary in the diffusion analysis is mapped from the reference space. Certain applications (like TBSS) and methodologies (like building the connectivity matrix by clustering the tracks) may not be performed correctly on the native (distorted) diffusion space because they still need a mapping to the undistorted space. Using regseg, the tracks obtained in native space can be unwarped using the resulting estimation of the deformation field. This methodological variation will be further investigated, to ensure which processing design yields the most accurate tractography results.

Beyond the presented application on dMRI data, regseg can be indicated in situations where there are precise surfaces delineating the structure, a target multivariate image in which the surfaces must be fitted, and the mapping between the surfaces and the volume encodes relevant physiological information, such as the normal/abnormal development or the macroscopic dynamics of organs and tissues. For instance, regseg may be applied in fields like neonatal brain image segmentation in longitudinal MRI studies of the early developmental patterns (Shi et al., 2010). In these studies, the surfaces obtained in a mature time point of the brain are retrospectively propagated to the initial time points, regardless of the changes in the contrast and spatial development between them. More generally, regseg may also be applied to the personalized study of longitudinal alteration of the brain using multispectral images, for instance in the case of traumatic brain injury (Irimia et al., 2014) or in monitoring brain tumors (Weizman et al., 2014).

\section{Conclusion}

Regseg is a variational framework for the simultaneous segmentation and registration of 3D dMRI data obtained from the human brain, where within-subject anatomical information is used as a reference. The registration method segments the target multivariate image into several competing regions, which are defined explicitly by their limiting surfaces. The surfaces are active and they evolve on a free-form deformation field supported by the B-spline basis. A descent optimization strategy is guided by shape gradients computed on the current partition of the target image. Regseg uses active contours without edges and it searches for homogeneous regions within the image. We tested regseg using digital phantoms by simulating T1w and T2w MRI warped with smooth and random deformations. The resulting misregistration of the contours was significantly lower than the image resolution of the phantoms.

We proposed regseg for simultaneously segmenting and registering dMRI data to their corresponding T1w image from the same subject. We demonstrated the accuracy of the proposed method based on visual assessments of the results obtained by regseg and cross-comparisons with a widely used technique. Moreover, regseg does not require any images in addition to the minimal acquisition protocol, which only utilizes T1w and dMRI. As well as the proposed application to dMRI data, other potential uses of regseg are atlas-based segmentation and tracking objects in time-series.

\section{Availability and reproducibility statement}

We considered the reproducibility of our results as a design requirement. Therefore, we used real data obtained from the Human Connectome Project (Van Essen et al., 2012) and all of the software utilized in this study is also publicly available. Regseg was implemented on top of ITK-4.6 (Insight Registration and Segmentation Toolkit, http:// www.itk.org). The evaluation instruments (Fig. 4) were implemented using nipype (Gorgolewski et al., 2011) to ensure their reproducibility. All of the research elements (data, source code, figures, manuscript sources, etc.) involved in this study are publicly available under a unique package (Esteban and Zosso, 2015).

\section{Author contributions}

All the authors contributed to this study. OE implemented the method, designed and conducted the experiments, wrote the paper, simulated the phantoms, and prepared the real data. DZ devised and drafted the registration method, generated early phantom datasets, and collaborated in the implementation of the method. $A D, M B C$, and $M J L C$ interpreted the results. $A D, M B C, M J L C, J P T$, and AS advised on all aspects of the study.

\section{Acknowledgments}

The authors thank Y. Alemán and G. Wollny for their thorough reviews of the manuscript, V. Estellers for early discussions at the beginning of this project, and $\mathrm{L}$. A. Vese for her support during OE's research visits to her laboratory. We also thank A. Leemans for kindly sharing a p-code version of ExploreDTI from which the settings for elastix could be extracted.

DZ was supported by the Swiss National Science Foundation under grants PBELP2-137727, P300P2-147778, and NSF-DMS 1418812. This study was supported by the Spanish Ministry of Science and Innovation (projects TEC-2013-48251-C2-2-R and TEC2015-66978-R), Comunidad de Madrid (TOPUS S2013/MIT-3024) and the European Regional Development Funds, the Center for Biomedical Imaging (CIBM) of the Geneva and Lausanne Universities and the EPFL, as well as the Leenaards Foundation and Louis Jeantet Foundation.

\section{Appendix A. Simplifying the regularization term}

The exponentials of the Thikonov regularization prior in Eq. (6) have the general form $\mathbf{v}^{T} \mathbf{M v}$. If $\mathbf{M}$ is a $n \times n$ diagonal matrix such that $\mathbf{M}=$ $\mathbf{m} \mathbf{I}_{n}$, then:

$\mathbf{v}^{T} \mathbf{M v}=\mathbf{m} \cdot\left(\mathbf{v}^{T} \mathbf{I}_{n} \mathbf{v}\right)=\mathbf{m} \cdot \mathbf{v}^{\cdot 2}$,

where we have introduced the Hadamard power notation. ${ }^{5}$

\footnotetext{
${ }^{5}$ The Hadamard power of a matrix or a vector is the power of its elements $\mathrm{M}^{-p}=\left(m_{i j}{ }^{p}\right)$.
} 
In general, the anisotropy of the distortion field is aligned with the voxel coordinate system, so $\mathbf{A}$ and $\mathbf{B}$ of Eq. (7) can be simplified to diagonal matrices to regularize the registration process, such that $\mathbf{A}=\boldsymbol{\alpha} \mathbf{I}_{n}$ and $\mathbf{B}=\boldsymbol{\beta} \mathbf{I}_{n}$. By substituting into Eq. (7), we obtain:

$$
E(\mathbf{u})=\text { Const. }+\sum_{l} \int_{\Omega_{l}} \mathcal{D}_{l}^{2}\left(\mathbf{f}^{\prime}\right) d \mathbf{r}+\int_{\Omega} \frac{1}{2}\left[\boldsymbol{\alpha} \cdot \mathbf{u}^{2}+\boldsymbol{\beta} \cdot(\nabla \mathbf{u})^{-2}\right] d \mathbf{r}
$$

\section{Appendix B. Application of the shape-gradients}

The computation of gradients at the locations of the active contours in the instant $t$ is based on the work of Herbulot et al. (2006). Let $F(\mathbf{r})$ be an "arbitrary" function over the image domain $\Omega=\Omega_{l} \cup \Omega_{m}$ split in two regions $l$ and $m$, and $\Gamma_{l, m}$ a closed boundary between them. We now derive the domain integral w.r.t. $t$ :

$\frac{\partial}{\partial t} \int_{\Omega} F(\mathbf{r}) d \mathbf{r}=\int_{\Omega} \frac{\partial}{\partial t} F(\mathbf{r}) d \mathbf{r}=\int_{\Gamma_{l, m}} F(\mathbf{r})\left\langle\frac{\partial \Gamma_{l, m}}{\partial t}, N_{\Gamma_{l, m}}\right\rangle d \mathbf{r}$,

where $\left\langle\frac{\partial \Gamma_{i, m}}{\partial t}, N_{\Gamma_{l, m}}\right\rangle$ is the projection of the boundary movement on the unit inward normal $N_{\Gamma_{l, m}}$. Assuming that the region descriptors $\left\{\boldsymbol{\mu}_{l}, \mathbf{\Sigma}_{l}\right\}$ vary slowly enough, we can consider that $\frac{\partial}{\partial t} F(\mathbf{r})=0$ and thus:

$\frac{\partial}{\partial t} \int_{\Omega} F(\mathbf{r}) d \mathbf{r}=-\int_{\Gamma_{l, m}} F(\mathbf{r})\left\langle\frac{\partial \Gamma_{l, m}}{\partial t}, N_{\Gamma_{l, m}}\right\rangle d \mathbf{r}$

The Eq. (A.3) is discretized as follows. First, the surface between limiting regions $l$ and $m\left(\Gamma_{l, m}\right)$ is explicitly represented by a discrete set of vertices $\mathbf{v}_{i}$, with $i \in\left\{0, \ldots, N_{p}-1\right\}$. Consequently, the inwards normal of the surface $N_{\Gamma_{l, m}}$ is represented by the discrete set of normals $\hat{\mathbf{n}}_{i}$ at each vertex of the mesh. The resulting summation is, therefore, discrete and the integral operator is replaced by the sum:

$$
\begin{aligned}
\frac{\partial}{\partial t} \int_{\Omega} F(\mathbf{r}) d \mathbf{r} & =\underbrace{\int_{\Omega} \frac{\partial}{\partial t} F(\mathbf{r}) d \mathbf{r}}_{\text {Functional's evolution }}-\int_{\Gamma_{l, m}} F(\mathbf{r})\left\langle\frac{\partial \Gamma_{l, m}}{\partial t}, N_{\Gamma_{l, m}}\right\rangle d \mathbf{r} \\
& =-\sum_{p} \frac{1}{A_{p}} \sum_{i} a_{i} F\left(\mathbf{v}_{i}\right)\langle\underbrace{\frac{\partial \mathbf{v}_{i}}{\partial t}, \hat{\mathbf{n}}_{i}}_{\text {speed of } \mathbf{v}_{i}}\rangle .
\end{aligned}
$$

where $a_{i}$ is the area corresponding to vertex $\mathbf{v}_{i}$, and $A_{p}=\sum_{i} a_{i}$ is the total area of the surface $p$. In the following, we will refer as $w_{p, i}=a_{i} / A_{p}$ to the area contribution of $\mathbf{v}_{i}$ to the total area of the surface it belongs to. For simplicity, the sum over $p$ can be also removed, as the vertices belong to only one of the total $P$ contours.

Then, the speed of $\mathbf{v}_{i}$ is discretized using the artificial time-step parameter $\delta$, as the displacement $\frac{\partial \mathbf{v}_{i}}{\partial t}=\mathbf{v}_{i}(\delta=t+1)-\mathbf{v}_{i}(\delta=t)$ :

$\frac{\partial}{\partial t} \int_{\Omega} F(\mathbf{r}) d \mathbf{r}=-\sum_{i} w_{p, i} F\left(\mathbf{v}_{i}\right) \frac{\partial v_{i}}{\partial t} \cdot \hat{\mathbf{n}}_{i}$.

Since the energy functional is defined over competing regions, the displacement of $\mathbf{v}_{i}$ will cause an energy exchange between the limiting regions, and therefore $F(\mathbf{r})$ must be split in two terms, $F_{i n}(\mathbf{r})$ corresponding to the interior region and $F_{\text {out }}(\mathbf{r})$ to the exterior:

$\frac{\partial}{\partial t} \int_{\Omega} F(\mathbf{r}) d \mathbf{r}=-\sum_{i} \frac{\partial \mathbf{v}_{i}}{\partial t} \cdot \underbrace{w_{p, i}\left[F_{\text {out }}\left(\mathbf{v}_{i}\right)-F_{\text {in }}\left(\mathbf{v}_{i}\right)\right] \hat{\mathbf{n}}_{i}}_{\bar{s}_{i} \text { in Figure 1 }}$.

Then, we identify the shape gradient contribution $\mathbf{g}_{k}$ on the coefficients $\mathbf{u}_{k}$ of the B-spline grid to obtain the definition of $\mathbf{g}_{k}$ given in
Eq. (12):

$$
\begin{aligned}
\mathbf{g}_{k} & =-\sum_{i}\left\langle\frac{\partial \mathbf{v}_{i}^{\prime}}{\partial \mathbf{u}_{k}}, \overline{s_{i}^{\prime}}\right\rangle \\
\text { with } \overline{s^{\prime}} & =w_{i}\left[\mathcal{D}_{\text {out }}^{2}\left(\mathbf{f}_{i}^{\prime}\right)\right]-\mathcal{D}_{\text {in }}^{2}\left(\mathbf{f}_{i}^{\prime}\right) \hat{\mathbf{n}}_{i}, \\
\text { and } \frac{\partial \mathbf{v}_{i}^{\prime}}{\partial \mathbf{u}_{k}} & =\frac{\partial}{\partial \mathbf{u}_{k}}\left\{\mathbf{v}_{i}+\sum_{k} \psi_{k}\left(\mathbf{v}_{i}\right) \mathbf{u}_{k}\right\}=\psi_{k}\left(\mathbf{v}_{i}\right) \hat{\mathbf{e}},
\end{aligned}
$$

where $\psi_{k}$ and $\mathbf{u}_{k}$ define our B-spline deformation model (Eq. (8)) and $\hat{\mathbf{e}}$ is the coordinates system's unit vector.

\section{References}

Andersson, J.L., Skare, S., Ashburner, J., 2003. How to correct susceptibility distortions in spin-echo echo-planar images: application to diffusion tensor imaging. Neurolmage 20 (2), 870-888. http://dx.doi.org/10.1016/S1053-8119(03)00336-7.

Andersson, J.L.R., Xu, J., Yacoub, E., Auerbach, E., Moeller, S., Ugurbil, K., 2012. A comprehensive Gaussian process framework for correcting distortions and movements in diffusion images. 20th ISMRM, p. 2426 (Melbourne, Australia).

Basser, P., Pierpaoli, C., 1996. Microstructural and physiological features of tissues elucidated by quantitative-diffusion-tensor MRI. J. Magn. Reson. B 111 (3), 209-219. http://dx.doi.org/10.1016/j.jmr.2011.09.022.

Caruyer, E., Daducci, A., Descoteaux, M., Houde, J.-C., Thiran, J.-P., Verma, R., 2014. Phantomas: a flexible software library to simulate diffusion MR phantoms. 23th ISMRM (Milano, Italy).

Chan, T.F., Vese, L.A., 2001. Active contours without edges. IEEE Trans. Image Process. 10 (2), 266-277. http://dx.doi.org/10.1109/83.902291.

Chiou, J.Y., Nalcioglu, O., 2000. A simple method to correct off-resonance related distortion in echo planar imaging. 8th ISMRM, p. 1711 (Denver, USA).

Commandeur, F., Velut, J., Acosta, O., 2011. A VTK algorithm for the computation of the Hausdorff distance. VTK J. 839.

Cordes, D., Arfanakis, K., Haughton, V., Meyerand, M., 2000. Geometric distortion correction in EPI using two images with orthogonal phase-encoding directions. 8th ISMRM, p. 1712 (Denver, USA)

Craddock, R.C., Jbabdi, S., Yan, C.-G., Vogelstein, J.T., Castellanos, F.X., Di Martino, A., Kelly, C., Heberlein, K., Colcombe, S., Milham, M.P., 2013. Imaging human connectomes at the macroscale. Nat. Methods 10 (6), 524-539. http://dx.doi.org/10.1038/nmeth. 2482.

Cuadra, M., Cammoun, L., Butz, T., Cuisenaire, O., Thiran, J.P., 2005. Comparison and validation of tissue modelization and statistical classification methods in $\mathrm{T} 1$ weighted MR brain images. IEEE Trans. Med. Imaging 24 (12), 1548-1565. http://dx.doi.org/ 10.1109/TMI.2005.857652

Daducci, A., Canales-Rodríguez, E.J., Zhang, H., Dyrby, T.B., Alexander, D.C., Thiran, J.-P., 2015. Accelerated Microstructure Imaging via Convex Optimization (AMICO) from diffusion MRI data. Neurolmage 105, 32-44. http://dx.doi.org/10.1016/j. neuroimage.2014.10.026

Ennis, D.B., Kindlmann, G., 2006. Orthogonal tensor invariants and the analysis of diffusion tensor magnetic resonance images. Magn. Reson. Med. 55 (1), 136-146. http:// dx.doi.org/10.1002/mrm.20741.

Esteban, O., Zosso, D., 2015. RegSeg: structure-informed segmentation and registration of brain MR images. Zenodo [Software] http://dx.doi.org/10.5281/zenodo.33282.

Esteban, O., Daducci, A., Caruyer, E., O'Brien, K., Ledesma-Carbayo, M.J., Bach-Cuadra, M Santos, A., 2014a. Simulation-based evaluation of susceptibility distortion correction methods in diffusion MRI for connectivity analysis. 11th ISBI, pp. 738-741 http:// dx.doi.org/10.1109/ISBI.2014.6867976 (Beijing, China).

Esteban, O., Wollny, G., Gorthi, S., Ledesma-Carbayo, M.-J., Thiran, J.-P., Santos, A., BachCuadra, M., 2014b. MBIS: Multivariate Bayesian Image Segmentation tool. Comput. Methods Prog. Biomed. 115 (2), 76-94. http://dx.doi.org/10.1016/j.cmpb.2014.03. 003.

Esteban, O., Zosso, D., Daducci, A., Bach-Cuadra, M., Ledesma-Carbayo, M.J., Thiran, J.-P. Santos, A., 2016. Data on the verification and validation of segmentation and registration methods for diffusion MRI (supplemental materials). Data in Brief, (in press) http://dx.doi.org/10.1016/j.dib.2016.06.049.

Fischl, B., 2012. FreeSurfer. Neurolmage 62 (2), 774-781. http://dx.doi.org/10.1016/j. neuroimage.2012.01.021.

Gass, T., Székely, G., Goksel, O., 2014. Simultaneous segmentation and multiresolution nonrigid atlas registration. IEEE Trans. Image Process. 23 (7), 2931-2943. http://dx doi.org/10.1109/TIP.2014.2322447.

Glasser, M.F., Sotiropoulos, S.N., Wilson, J.A., Coalson, T.S., Fischl, B., Andersson, J.L., Xu, J., Jbabdi, S., Webster, M., Polimeni, J.R., Van Essen, D.C., Jenkinson, M., 2013. The minimal preprocessing pipelines for the human connectome project. Neurolmage 80 105-124. http://dx.doi.org/10.1016/j.neuroimage.2013.04.127.

Gorgolewski, K., Burns, C.D., Madison, C., Clark, D., Halchenko, Y.O., Waskom, M.L., Ghosh, S., 2011. Nipype: a flexible, lightweight and extensible neuroimaging data processing framework in python. Front. Neuroinform. 5, 13. http://dx.doi.org/10.3389/fninf 2011.00013.

Gorthi, S., Duay, V., Bresson, X., Bach Cuadra, M., Sánchez Castro, F.]., Pollo, C., Allal, A.S. Thiran, J.-P., 2011. Active deformation fields: dense deformation field estimation for atlas-based segmentation using the active contour framework. Med. Image Anal. 15 (6), 787-800. http://dx.doi.org/10.1016/j.media.2011.05.008. 
Greve, D.N., Fischl, B., 2009. Accurate and robust brain image alignment using boundarybased registration. Neurolmage 48 (1), 63-72. http://dx.doi.org/10.1016/j. neuroimage.2009.06.060.

Hadjiprocopis, A., Rashid, W., Tofts, P.S., 2005. Unbiased segmentation of diffusionweighted magnetic resonance images of the brain using iterative clustering. Magn. Reson. Imaging 23 (8), 877-885. http://dx.doi.org/10.1016/j.mri.2005.07.010.

Hagmann, P., Cammoun, L., Gigandet, X., Meuli, R., Honey, C.J., Wedeen, V.J., Sporns, O., 2008. Mapping the structural core of human cerebral cortex. PLoS Biol. 6 (7), e159. http://dx.doi.org/10.1371/journal.pbio.0060159.

Han, D., Singh, V., Lee, J., Zakszewski, E., Adluru, N., Oakes, T., Alexander, A., 2009. An experimental evaluation of diffusion tensor image segmentation using graph-cuts. 31th EMBC, pp. 5653-5656 http://dx.doi.org/10.1109/IEMBS.2009.5333767 (Minneapolis, Minnesota, USA).

Herbulot, A., Jehan-Besson, S., Duffner, S., Barlaud, M., Aubert, G., 2006. Segmentation of vectorial image features using shape gradients and information measures. J. Math. Imaging Vission 25 (3), 365-386. http://dx.doi.org/10.1007/s10851-006-6898-y.

Holland, D., Kuperman, J.M., Dale, A.M., 2010. Efficient correction of inhomogeneous static magnetic field-induced distortion in Echo Planar Imaging. Neurolmage 50 (1) 175-183. http://dx.doi.org/10.1016/j.neuroimage.2009.11.044.

Irfanoglu, M.O., Walker, L., Sammet, S., Pierpaoli, C., Machiraju, R., 2011. Susceptibility distortion correction for echo planar images with non-uniform B-spline grid sampling: diffusion tensor image study. 14th MICCAI, LNCS 6892. Springer, Berlin Heidelberg pp. 174-181 http://dx.doi.org/10.1007/978-3-642-23629-7_22 (Toronto, Canada).

Irfanoglu, M.O., Modi, P., Nayak, A., Hutchinson, E.B., Sarlls, J., Pierpaoli, C., 2015. DRBUDDI (Diffeomorphic Registration for Blip-Up Blip-Down Diffusion Imaging) method for correcting echo planar imaging distortions. Neurolmage 106, 284-299. http:/ dx.doi.org/10.1016/j.neuroimage.2014.11.042.

Irimia, A., Goh, S.-Y.M., Torgerson, C.M., Vespa, P.M., Van Horn, J.D., 2014. Structural and connectomic neuroimaging for the personalized study of longitudinal alterations in cortical shape, thickness and connectivity after traumatic brain injury. J. Neurosurg. Sci. 58 (3), 129-144.

Jehan-Besson, S., Barlaud, M., Aubert, G., 2003. DREAM2S: deformable regions driven by an Eulerian accurate minimization method for image and video segmentation. Int. J. Comput. Vis. 53 (1), 45-70. http://dx.doi.org/10.1023/A:1023031708305

Jeurissen, B., Tournier, J.-D., Dhollander, T., Connelly, A., Sijbers, J., 2014. Multi-tissue constrained spherical deconvolution for improved analysis of multi-shell diffusion MRI data. Neurolmage 103, 411-426. http://dx.doi.org/10.1016/j.neuroimage.2014. 07.061 .

Jeurissen, B., Tournier, J-D., Sijbers, J., 2015. Tissue-type segmentation using non-negative matrix factorization of multi-shell diffusion-weighted MRI images. 23th ISMRM, p. 0346 (Toronto, Canada).

Jezzard, P., Balaban, R.S., 1995. Correction for geometric distortion in echo planar images from B0 field variations. Magn. Reson. Med. 34 (1), 65-73. http://dx.doi.org/10.1002 mrm.1910340111.

Jonasson, L., 2005. Segmentation of Diffusion Weighted MRI Using the Level Set Framework (Ph.D. Thesis) École Polytechnique Fédérale de Lausanne, Lausanne (Swizerland).

Klein, S., Staring, M., Murphy, K., Viergever, M., Pluim, J.P.W., 2010. Elastix: a toolbox for intensity-based medical image registration. IEEE Trans. Med. Imaging 29 (1), 196-205. http://dx.doi.org/10.1109/TMI.2009.2035616.

Kybic, J., Thevenaz, P., Nirkko, A., Unser, M., 2000. Unwarping of unidirectionally distorted EPI images. IEEE Trans. Med. Imaging 19 (2), 80-93. http://dx.doi.org/10.1109/42 836368.

Le Guyader, C., Vese, L.A., 2011. A combined segmentation and registration framework with a nonlinear elasticity smoother. Comput. Vis. Image Underst. 115 (12) 1689-1709. http://dx.doi.org/10.1016/j.cviu.2011.05.009.

Leemans, A., Jeurissen, B., Sijbers, J., Jones, D., 2009. ExploreDTI: a graphical toolbox for processing, analyzing, and visualizing diffusion MR data. 17th ISMRM, p. 3537 (Hawaii, USA).

Liu, T., Li, H., Wong, K., Tarokh, A., Guo, L., Wong, S.T., 2007. Brain tissue segmentation based on DTI data. Neurolmage 38 (1), 114-123. http://dx.doi.org/10.1016/j. neuroimage.2007.07.002

Lu, C.-F., Wang, P.-S., Chou, Y.-C., Li, H.-C., Soong, B.-W., Wu, Y.-T., 2008. Segmentation of diffusion-weighted brain images using expectation maximization algorithm initialized by hierarchical clustering. 30th EMBS, pp. 5502-5505 http://dx.doi.org/10. 1109/IEMBS.2008.4650460 (Vancouver, Canada).
Mori, S., Crain, B.J., Chacko, V.P., Van Zijl, P.C.M., 1999. Three-dimensional tracking of axonal projections in the brain by magnetic resonance imaging. Ann. Neurol. 45 (2), 265-269. http://dx.doi.org/10.1002/1531-8249(199902)45:2<265::AID-ANA21>3.0. $\mathrm{CO} ; 2-3$.

Nagel, H.-H., Enkelmann, W., 1986. An investigation of smoothness constraints for the estimation of displacement vector fields from image sequences. IEEE Trans. Pattern Anal. Mach. Intell. 8 (5), 565-593. http://dx.doi.org/10.1109/TPAMI.1986.4767833.

Paragios, N., 2003. A level set approach for shape-driven segmentation and tracking of the left ventricle. IEEE Trans. Med. Imaging 22 (6), 773-776. http://dx.doi.org/10.1109/ TMI.2003.814785

Pohl, K.M., Fisher, J., Grimson, W.E.Le, Kikinis, R., Wells, W.M., 2006. A Bayesian model for joint segmentation and registration. Neurolmage 31 (1), 228-239. http://dx.doi.org/ 10.1016/j.neuroimage.2005.11.044.

Rousson, M., Lenglet, C., Deriche, R., 2004. Level set and region based surface propagation for diffusion tensor MRI segmentation. 8th ECCV, INCS 3117. Springer, Berlin Heidelberg, pp. 123-134 http://dx.doi.org/10.1007/978-3-540-27816-0_11 (Prague, Czech Republic).

Rueckert, D., Aljabar, P., Heckemann, R.A., Hajnal, J.V., Hammers, A., 2006. Diffeomorphic registration using B-splines. 9th MICCAI, LNCS 4191. Springer, Berlin Heidelberg, pp. 702-709 http://dx.doi.org/10.1007/11866763 86 (Copenhagen, Denmark).

Shi, F., Fan, Y., Tang, S., Gilmore, J.H., Lin, W., Shen, D., 2010. Neonatal brain image segmentation in longitudinal MRI studies. Neurolmage 49 (1), 391-400. http://dx.doi.org/10. 1016/j.neuroimage.2009.07.066.

Smith, R.E., Tournier, J.-D., Calamante, F., Connelly, A., 2012. Anatomically-constrained tractography: improved diffusion MRI streamlines tractography through effective use of anatomical information. NeuroImage 62 (3), 1924-1938. http://dx.doi.org/ 10.1016/j.neuroimage.2012.06.005.

Studholme, C., Constable, R., Duncan, J., 2000. Accurate alignment of functional EPI data to anatomical MRI using a physics-based distortion model. IEEE Trans. Med. Imaging 19 (11), 1115-1127. http://dx.doi.org/10.1109/42.896788.

Taoka, T., Morikawa, M., Akashi, T., Miyasaka, T., Nakagawa, H., Kiuchi, K., Kishimoto, T., Kichikawa, K., 2009. Fractional anisotropy-threshold dependence in tract-based diffusion tensor analysis: evaluation of the uncinate fasciculus in Alzheimer disease. AJNR Am. J. Neuroradiol. 30 (9), 1700-1703. http://dx.doi.org/10.3174/ajnr.A1698.

Tournier, J.-D., Calamante, F., Connelly, A., 2012. MRtrix: diffusion tractography in crossing fiber regions. Int. J. Imaging Syst. Technol. 22 (1), 53-66. http://dx.doi.org/10.1002/ ima.22005.

Tustison, N.J., Johnson, H.J., Rohlfing, T., Klein, A., Ghosh, S.S., Ibanez, L., Avants, B.B., 2013. Instrumentation bias in the use and evaluation of scientific software: recommendations for reproducible practices in the computational sciences. Front. Neurosci. 7 (162). http://dx.doi.org/10.3389/fnins.2013.00162

Van Essen, D., Ugurbil, K., Auerbach, E., Barch, D., Behrens, T., Bucholz, R., Chang, A., Chen, L Corbetta, M Curtiss, S, Della Penna, S, Feinberg D, Glasser, M. Harel, N., Heath, A. Larson-Prior, L., Marcus, D., Michalareas, G., Moeller, S., Oostenveld, R., Petersen, S., Prior, F., Schlaggar, B., Smith, S., Snyder, A., Xu, J., Yacoub, E., 2012. The human connectome project: a data acquisition perspective. Neurolmage 62 (4), 2222-2231. http://dx.doi.org/10.1016/j.neuroimage.2012.02.018.

Van Leemput, K., Maes, F., Vandermeulen, D., Suetens, P., 1999. Automated model-based tissue classification of MR images of the brain. IEEE Trans. Med. Imaging $18(10)$, 897-908. http://dx.doi.org/10.1109/42.811270.

Weizman, L., Sira, L.B., Joskowicz, L., Rubin, D.L., Yeom, K.W., Constantini, S., Shofty, B., Bashat, D.B., 2014. Semiautomatic segmentation and follow-up of multicomponent low-grade tumors in longitudinal brain MRI studies. Med. Phys. 41 (5), 052303. http://dx.doi.org/10.1118/1.4871040.

Wyatt, P.P., Noble, J., 2003. MAP MRF joint segmentation and registration of medical images. Med. Image Anal. 7 (4), 539-552. http://dx.doi.org/10.1016/\$13618415(03)00067-7.

Yezzi, A., Zöllei, L, Kapur, T., 2003. A variational framework for integrating segmentation and registration through active contours. Med. Image Anal. 7 (2), 171-185. http://dx. doi.org/10.1016/S1361-8415(03)00004-5.

Zhukov, L., Museth, K., Breen, D., Whitaker, R., Barr, A., 2003. Level set modeling and segmentation of DT-MRI brain data. J. Electron. Imaging 12 (1), 125-133. http://dx.doi. org/10.1117/1.1527628. 